\title{
1 Clinical recovery of Macaca fascicularis infected with Plasmodium knowlesi
}

2 Mariko S. Peterson ${ }^{\dagger 1,2}$, Chester J. Joyner ${ }^{\dagger 1,2,3}$, Jessica A. Brady ${ }^{4}$, Jennifer S. Wood ${ }^{5}$, Monica Cabrera-

3 Mora $^{1,2}$, Celia L. Saney ${ }^{1,2}$, Luis L. Fonseca ${ }^{6}$, Wayne T.Cheng ${ }^{3}$, Jianlin Jang ${ }^{1,2}$, Stacey A. Lapp ${ }^{1,2}$,

4 Stephanie R. Soderberg ${ }^{1,2}$, Mustafa V. Nural ${ }^{7}$, Jay C. Humphrey ${ }^{7}$, Allison Hankus ${ }^{1,2}$, Deepa Machiah ${ }^{8}$,

5 Ebru Karpuzoglu ${ }^{1,2}$, Jeremy D. DeBarry ${ }^{7}$, MaHPIC-Consortium ${ }^{1,2}$, Rabindra Tirouvanziam ${ }^{9}$, Jessica C.

6 Kissinger ${ }^{7,10,11}$, Alberto Moreno ${ }^{1,2,12}$, Sanjeev Gumber ${ }^{8,13}$, Eberhard O. Voit ${ }^{6}$, Juan B. Gutiérrez ${ }^{14}$, Regina

7 Joice Cordy ${ }^{1,2}$ and Mary R. Galinski ${ }^{1,2,12}$

\section{These authors contributed equally to this work}

$9{ }^{1}$ Yerkes National Primate Research Center, Emory University, Atlanta, GA, USA

$10{ }^{2}$ Emory Vaccine Center, Emory University, Atlanta, GA, USA

$11{ }^{3}$ Center for Vaccines and Immunology, Department of Infectious Diseases, University of Georgia, Athens

12 GA, USA

$13{ }^{4}$ School of Chemical, Materials and Biomedical Engineering, University of Georgia, Athens, GA, USA

$14{ }^{5}$ Division of Animal Resources, Yerkes National Primate Research Center, Emory University, Atlanta,

15 GA, USA

$16{ }^{6}$ The Wallace H. Coulter Department of Biomedical Engineering, Georgia Institute of Technology and

17 Emory University, Atlanta, GA, USA

$18{ }^{7}$ Institute of Bioinformatics, University of Georgia, Athens, GA, USA

$19{ }^{8}$ Division of Pathology, Yerkes National Primate Research Center, Atlanta, GA, USA

$20{ }^{9}$ Department of Pediatrics, Emory University School of Medicine, Atlanta, GA, USA

$21{ }^{10}$ Department of Genetics, University of Georgia, Athens, GA, USA

$22{ }^{11}$ Center for Tropical and Emerging Global Diseases, University of Georgia, Athens, GA, USA

$23{ }^{12}$ Division of Infectious Diseases, Department of Medicine, Emory University School of Medicine,

24 Atlanta, GA

$25{ }^{13}$ Department of Pathology and Laboratory Medicine, Emory School of Medicine, Atlanta, GA

$26{ }^{14}$ Department of Mathematics, University of Texas at San Antonio, San Antonio, TX, USA 


\section{Current Addresses}

30 Mariko S. Peterson: Emory University School of Medicine, Atlanta, GA, USA

31 Chester J. Joyner: Center for Vaccines and Immunology, Department of Infectious Diseases, College of

32 Veterinary Medicine, University of Georgia, Athens GA, USA

33 Jessica A. Brady: Eli Lilly and Company, Indianapolis, IN, USA

34 Monica Cabrera-Mora: Emory University School of Medicine, Atlanta, GA, USA

35 Luis L. Fonseca: Division of Pulmonary, Critical Care, and Sleep Medicine, Department of Medicine,

36 College of Medicine, University of Florida, Gainesville, FL, USA

37 Stacey A. Lapp: Emory University School of Medicine, Atlanta, GA, USA

38 Stephanie R. Soderberg: Thermo Fisher Scientific, South San Francisco, CA, USA

39 Jay Humphrey: Center for Tropical \& Emerging Global Diseases, University of Georgia, Athens, GA,

40 USA

41 Allison Hankus: The MITRE Corporation, Atlanta, GA, USA

42 Ebru Karpuzoglu: Department of Endocrinology, Atlanta VA Medical Center, Decatur, GA, USA

43 Jeremy D. DeBarry: Center for Topical and Emerging Global Diseases, University of Georgia, Athens, 44 GA, USA

45 Juan B. Gutierrez: Department of Mathematics, University of Texas at San Antonio, San Antonio, TX, 46 USA

47 Regina Joice Cordy: Department of Biology, Wake Forest University, Winston-Salem, NC, USA

50 *Corresponding Author: Mary R. Galinski, Emory Vaccine Center, Yerkes National Primate Research

51 Center, 954 Gatewood Rd, Atlanta, GA 30329. Tel: 404-727-7214. Email: Mary.Galinski@emory.edu

53 Competing Intereststs: The authors have declared that no conflict of interest exists. 


\section{ABSTRACT}

\section{Background}

57 Kra monkeys (Macaca fascicularis), a natural host of Plasmodium knowlesi, control

58 parasitaemia caused by this parasite species and escape death without treatment. Knowledge of

59 the disease progression and resilience in kra monkeys will aid the effective use of this species to

60 study mechanisms of resilience to malaria. This longitudinal study aimed to define clinical,

61 physiological and pathological changes in kra monkeys infected with P. knowlesi, which could

62 explain their resilient phenotype.

\section{Methods}

64 Kra monkeys ( $n=15$, male, young adults) were infected intravenously with cryopreserved $P$.

65 knowlesi sporozoites and the resulting parasitaemias were monitored daily. Complete blood

66 counts, reticulocyte counts, blood chemistry and physiological telemetry data $(n=7)$ were

67 acquired as described prior to infection to establish baseline values and then daily after

68 inoculation for up to 50 days. Bone marrow aspirates, plasma samples, and 22 tissue samples

69 were collected at specific time points to evaluate longitudinal clinical, physiological and

70 pathological effects of $P$. knowlesi infections.

\section{$71 \quad$ Results}

72 As expected, the kra monkeys controlled parasitaemia and remained with low-level, persistent

73 parasitaemias without antimalarial intervention. Unexpectedly, early in the infection, fevers

74 developed, which ultimately returned to baseline, as well as mild to moderate

75 thrombocytopaenia, and moderate to severe anaemia. Mathematical modeling and the

76 reticulocyte production index indicated that the anaemia was largely due to the removal of

77 uninfected erythrocytes and not impaired production of erythrocytes. Mild tissue damage was 
78 observed, and tissue parasite load was associated with tissue damage even though parasite

79 accumulation in the tissues was generally low.

80 Conclusions

81 Kra monkeys experimentally infected with P. knowlesi sporozoites presented with multiple

82 clinical signs of malaria that varied in severity among individuals. Overall, the animals shared

83 common mechanisms of resilience characterized by controlling parasitaemia 3-5 days after

84 patency, and controlling fever, coupled with physiological and bone marrow responses to

85 compensate for anaemia. Together, these responses likely minimized tissue damage while

86 supporting the establishment of chronic infections, which may be important for transmission in

87 natural endemic settings. These results provide new foundational insights into malaria

88 pathogenesis and resilience in kra monkeys, which may improve understanding of human

89 infections.

90

91

92

93

94

95

96

97

98

99 KEYWORDS 
bioRxiv preprint doi: https://doi.org/10.1101/2021.06.28.448877; this version posted June 28, 2021. The copyright holder for this preprint (which was not certified by peer review) is the author/funder. All rights reserved. No reuse allowed without permission.

100 Malaria; nonhuman primate models; infectious diseases; resilience; telemetry; fever;

101 haematology; anaemia, thrombocytopaenia, erythropoietin; bone marrow; histopathology

102 


\section{BACKGROUND}

Plasmodium knowlesi malaria cases have been identified in 10 of the 11 countries that comprise Southeast Asia; the one exception being Timor-Leste [reviewed in 1, 2, 3]. Since its recognition in 2004 in Malaysia as an emergent zoonotic parasite species [4], P. knowlesi has in fact risen as the major cause of human clinical cases of malaria in Malaysia and threatens to malaria were reported in 2018 [9]. Plasmodium knowlesi transmission to humans has been attributed to spillover events from peri-domestic infected macaques in village settings, or from

111 infected wild macaques in the setting of jungle trekking, foraging, farming, and logging activities

$112[1,5,10-13]$. At least three macaque species are endemic to these geographical regions and can

113 serve as reservoirs for P. knowlesi: Macaca fascicularis (the kra monkey, or long-tailed 114 macaque), Macaca nemestrina (pig-tailed macaque) and Macaca arctoides (stump-tailed 115 macaque) [14-17].

Plasmodium knowlesi infections cause symptoms that are generally associated with

117 malaria caused by other Plasmodium species (Plasmodium falciparum, Plasmodium vivax,

118 Plasmodium malariae, and Plasmodium ovale, and the closely related zoonotic species

119 Plasmodium cynomolgi) [18-21], including cyclical episodes of fever and chills with rigor.

120 Clinical complications have the potential to become exacerbated quickly given this species'

121 unique 24-hour replication cycle within the erythrocyte host cell [1, 5, 22-24]. Asymptomatic $P$.

122 knowlesi infections are less studied, but they have been shown to be common, including a high

123 percentage in children [12]. Clinically, thrombocytopaenia has been reported as a universal

124 problem with $P$. knowlesi infections $(<150,000$ platelets $/ \mu 1)$, with about $1 / 3$ of adult patients

125 showing severe thrombocytopaenia $(<50,000$ platelets/ $\mu l)$ [reviewed in 1$]$. Anaemia is not a 
126 predominant problem with $P$. knowlesi malaria in adults, though moderate anaemia (defined as

127 less than $10 \mathrm{~g} / \mathrm{dL}$ ) and recovery from this disease state have been observed, and some cases of

128 severe anaemia (less than $7 \mathrm{~g} / \mathrm{dL}$ ) have been documented [24, 25] and reviewed in [1]. In

129 contrast, anaemia has been identified as a predominant feature in children with P. knowlesi

130 malaria [26], despite children having lower parasitaemias (and no fatalities reported) [27]. While

131 hyperparasitaemia is associated with severe disease, it should be noted that $P$. knowlesi can cause

132 clinical manifestations at lower parasitaemias than other species (e.g., baseline median values on

133 hospital admission in Kapit of 1,387 P. knowlesi parasites/ $\mu$ l, compared to 4,258 $P$. vivax

134 parasites $/ \mu$ l and 26,781 P. falciparum parasites/ $\mu$ l) [24]. Also, Barber and others showed in a

135 prospective study that in Saba, Malaysia, P. knowlesi was more likely than P. falciparum to

136 cause severe disease [25].

137 In severe cases of $P$. knowlesi malaria, clinical signs and symptoms in adults have

138 included abdominal pain, shortness of breath, productive cough, and respiratory distress [1, 6,

$13923,24]$. Severe cases have included jaundice, acute kidney injury, metabolic acidosis, acute lung

140 injury, and shock, among others [reviewed in 28, 29]. High parasitaemia (>20,000 P. knowlesi

141 parasites $/ \mu \mathrm{l}$ ) along with jaundice and thrombocytopaenia have been identified as putative

142 indicators for severe disease $[1,24,25]$. Cerebral malaria and coma have not been associated

143 with $P$. knowlesi, although severe malaria including hyperparasitaemia can result in cerebral

144 pathology as shown in one reported autopsy case of P. knowlesi [23].

145 Daneshvar and colleagues reported close to $10 \%$ of hospital cases of $P$. knowlesi malaria

146 with severe complications and a case fatality rate in their study of $1.8 \%$ [24] while others have

147 reported higher numbers for severe cases (29\% and 39\% of hospital cases studied) [25, 30] and

148 deaths (six deaths out of 22 patients with a severe case of $P$. knowlesi malaria) [30]. As of 2017, 
14941 deaths were reported in Malaysia [31]. Notably, deaths are being averted in recent years with

150 improved monitoring and diagnosis and the timely use of intravenous artesunate [32] or

151 artemisinin combination therapies (ACTs) [25, 28, 33, 34].

152 As this zoonotic disease remains prevalent, gaining an improved understanding of the 153 pathogenesis of $P$. knowlesi is important. Such an understanding can be gained with the use of 154 nonhuman primate (NHP) models [reviewed in 35, 36, 37]. Experimental infections can be 155 designed that consider the infecting parasite species and genotype(s), the parasite stage and size 156 of the inoculum, the duration of infections, experimental interventions, treatments, and the 157 immune status of the host. Furthermore, tissue samples can be collected for pathology studies. Multiple NHP species can be experimentally infected with P. knowlesi [reviewed in 35, 159 36, 38]. The most explored have been rhesus and kra monkeys. Malaria-naïve rhesus macaques 160 will invariably succumb to $P$. knowlesi infection, unless treated with anti-malarial drugs, because 161 of the unceasing, overwhelming rise in parasitaemia and the continued cyclical destruction of 162 erythrocytes that occurs unabated in this species with the multiplication of the parasites every 24 163 hours [38-43]. Thus, rhesus macaques are viable animal models for severe disease due to 164 hyperparasitaemia. In the course of $\sim 50$ years, rhesus monkey infections became the main 165 experimental host to study $P$. knowlesi biology, virulence, immune responses, antigenic variation 166 and pathology, with much knowledge yet to be uncovered in each of these areas [e.g., see 41, 42$16745]$ and [reviewed in 36, 37]. In stark contrast, malaria-naïve kra monkeys are able to control 168 parasitaemia without antimalarial treatment, typically with a maximum of 1-3\% parasitaemia 169 [38-40, 46, 47], making them the preferred animal model for mild, moderate or chronic $P$. 170 knowlesi malaria. 
Despite the potential of kra monkeys for revealing mechanisms of resilience to $P$.

172 knowlesi infections, only a few small studies have so far reported features of $P$. knowlesi

173 infections and pathology in kra monkeys [48, 49]. Anderios and colleagues reported

174 haematological and liver and spleen histopathology data from two kra monkeys after 60 or 90

175 days of infection post-inoculation either with $P$. knowlesi-infected blood from a patient or

176 cryopreserved P. knowlesi (Malayan strain)-infected rhesus blood [48]. Barber and colleagues

177 studied the deformability of RBCs from both $P$. knowlesi-infected patients and three infected kra

178 monkeys [49]. In-depth longitudinal characterizations of clinical and other parameters during the

179 course of $P$. knowlesi infection in this resilient host species and tissue pathology analysis will

180 complement clinical investigations and therapeutic considerations.

181 By combining telemetric, clinical, parasitological and pathological data, collected

182 longitudinally for up to 50 days, the present investigation demonstrates that kra monkeys develop

183 and recover without treatment from clinical manifestations of malaria, including fever,

184 thrombocytopaenia and anaemia. Low parasitaemias, low parasite tissue burdens, and overall

185 mild tissue damage and organ dysfunction define this resilient phenotype. By highlighting the

186 clinical and pathological consequences of $P$. knowlesi, this study provides a framework for basic

187 and systems biological studies utilizing the $P$. knowlesi - kra monkey model for studying malaria

188 resilience and pathogenesis (Gupta et al., submitted). Possible systemic mechanisms and 189 potential research directions are discussed to better understand these phenomena and consider 190 future novel therapies. 
MATERIALS AND METHODS

197 Animal use. Experiments involving NHPs were performed at the Yerkes National Primate

198 Research Center (YNPRC), an AAALAC International-accredited facility, following ARRIVE

199 Guidelines and Recommendations [50]. All experimental, surgical, and necropsy procedures

200 were approved by Emory's IACUC and the Animal Care and Use Review Office (ACURO) of

201 the US Department of Defense and followed accordingly. The Emory's IACUC approval number

202 was PROTO201700484 - YER-2003344-ENTRPR-A. 15 M. fascicularis (Mauritius origin, born

203 and raised at the Mannheimer Foundation, Inc., Homestead, FL, USA), all healthy and malaria-

204 naïve young adults (3-5 years old) were assigned within three cohorts for sequential longitudinal

205 systems biology infection experiments, primarily aiming to understand the resilience of $M$.

206 fascicularis to P. knowlesi blood-stage infections (Supplemental Table 1 and Supplemental Figs.

207 1-3). Male monkeys were selected to eliminate confounding anaemia measurements due to

208 menstruation. All animals were housed socially, with 12-h light-dark cycles, in housing

209 compliant with the Animal Welfare Act and the Guide for the Care and Use of Laboratory

210 Animals. Environmental enrichment consisting of food and physical manipulanda was provided

211 daily. The animals received positive reinforcement training to habituate them to ear-stick blood

212 collections for blood smear preparations and clinical analyses requiring blood collections of less

213 than $150 \mu \mathrm{l}$. The weight of the animals was determined to be between $5.6 \mathrm{~kg}$ and $11.7 \mathrm{~kg}$ at the

214 endpoint of each experiment, when necropsies and tissue analyses were performed. The animals

215 were anaesthetized using ketamine and euthanized via intravenous administrations of 
216 barbiturates. This euthanasia method is an acceptable method of euthanasia for NHPs per the

217 recommendations of the "AVMA Guidelines for the Euthanasia of Animals".

219 Parasite isolates and inoculations. The monkeys from experimental cohorts E07, E33 and E35

220 (Supplemental Table 1) were infected intravenously with $\sim 100,000$ cryopreserved P. knowlesi

221 clone Pk1(A+) sporozoites derived from the Malayan strain of P. knowlesi [51] (kindly provided

222 by John W. Barnwell, Centers for Disease Control and Prevention, Atlanta, GA), which had been

223 suspended in RPMI 1640 with $50 \%$ fetal calf serum and quick frozen, and when thawed

224 estimated to be $0.1-1 \%$ viable; John W. Barnwell, personal communication). The Experiment 07

225 cohort was initially inoculated with sporozoites freshly dissected from mosquitoes at the Centers

226 for Disease Control and Prevention, but for unexplained reasons parasitaemia did not develop in

227 the blood. The E07 cohort was subsequently inoculated (about 80 days later) with the same batch

228 of cryopreserved sporozoites used to infect the two other cohorts. There was no evidence that the

229 animals had developed immunity based on similar infection kinetics across the cohorts, and

230 significant differences were not detected in the repeated baseline transcriptomes (Gupta et al.

231 submitted). Tissues from normal uninfected animals from E34, as well as archived tissue blocks

232 from the YNPRC tissue repository, were used as negative controls for histopathological

233 comparisons.

235 Sample collections. BM aspirates from the iliac crest and venous blood collections were 236 collected into EDTA. Capillary blood samples were collected into EDTA using standardized ear-

237 stick procedures for CBCs and monitoring of parasitaemias. 
239 Telemetry. The monkeys in the E07 cohort ( $n=7$, Supplementary Table 1$)$ had telemetry devices

240 surgically implanted prior to infection, for real-time monitoring of temperature and other vital

241 signs (Brady et al., manuscript in preparation). These devices were not included in the

242 experimental design of the E33 and E35 protocols, which comprised two iterative studies without

243 these measurements among the goals. In brief, the PhysioTelTM L11 telemetry implant device

244 was secured between the external and internal abdominal oblique muscles. Raw temperature

245 data, used in the current study, was obtained with a 1 hertz sampling frequency after which

246 missing value indicators were removed. Hourly temperature averages were obtained using a

247 subset of the data, minute averages obtained from the first 15 seconds of each minute if

248 available, and then smoothed using the Hodrick-Prescott filter to reduce noise. The normal

249 temperature range for each monkey was defined as the range of temperatures measured prior to

250 inoculation of $P$. knowlesi sporozoites. The febrile threshold was determined by considering the

251 parasitaemia prior to the time when the temperature rose above the individual's threshold. The

252 time-to-temperature response was determined as the day post-inoculation when the temperature

253 rose above the individual's threshold.

255 Quantification of reticulocyte production index. Reticulocyte production index was calculated to assess

256 bone marrow responsiveness to anaemia, and is given by the following equation, as described previously

257 [52]:

$$
R P I=\frac{\text { Percent peripheral reticulocytes }}{\text { Reticulocyte maturation }} * \frac{\text { Hemoglobin }}{\text { Individual baseline hemoglobin }}
$$

259 Parasite enumeration. Parasitaemia was enumerated daily between 1 PM and 3 PM when 260 synchronous ring-stage parasites predominated until the infections became patent. After patency, 
261 twice-daily capillary samples (8 AM, schizonts; and 1-3 PM ring stages) were acquired for

262 parasitaemia monitoring. Thick and thin blood smears were prepared and stained with Wright's

263 Giemsa. Thick blood smears were used to enumerate parasitaemias below $1 \%$ by the Earle Perez

264 method [53]. Once parasitaemia was greater than 1\%, thin blood smears were used for

265 parasitaemia measurements by determining the number of iRBCs out of 1,000 total RBCs. The

266 number of parasites per microliter of blood was calculated from thin blood smears by

267 multiplying the percentage of infected RBCs by the number of RBCs per microliter of blood

268 obtained from the CBCs. Cumulative parasitaemia was calculated for each animal by adding

269 together the daily parasitaemia (parasites/ $\mu 1$ ) from the day of inoculation to the day of necropsy.

270 Mean parasitaemia across five-day increments was calculated for each individual by defining 5-

271 day windows starting from the day of inoculation and averaging the parasitaemias within each

272 window. Subsequent calculations with haemoglobin levels and platelet counts were likewise

273 performed in this manner.

275 Quantification of parasite replication rate. The replication rate of parasites was calculated as

276 described previously [54]. Only the first peak was considered because it had the most robust 277 sample size.

279 Tissue collection, preservation, and pathology analysis. Tissues were collected at necropsy

280 from 22 organs from all longitudinal infection experiments with the exception of E33, which had

281 an abbreviated tissue collection, restricted to liver, lung, kidney, spleen, adrenal gland, BM,

282 stomach, duodenum, jejunum, and colon where previous pathology had been observed. Gross

283 lesions were photographed, and tissue sections were collected for histopathology. Each was 
preserved in $10 \%$ neutral buffered formalin, embedded in paraffin, sectioned at $4 \mu \mathrm{m}$, and stained with H\&E. Diagnostic characterization and scoring of histopathology were obtained from examination of randomized, blinded H\&E-stained tissues. Tissues were scored from 1 (low) to 4 (high) based on: inflammation, oedema, necrosis, haemorrhage, hyperplasia, fibrosis, and vasculitis. The scores were summed and whole organ scores obtained for comparison, as described previously with $P$. vivax infected S. boliviensis tissues [55].

iRBC quantification within tissues. $\mathrm{R} B \mathrm{RC}$ densities in the tissues were determined by counting the number of iRBCs in ten HPFs (1000x) under oil immersion on a standard light microscope,

293 as described previously with $P$. vivax infected S. boliviensis tissues [55]. All sections were 294 randomized and blinded.

Erythroid progenitor measurements by flow cytometry. Five microliters of BM aspirate were

297 placed into a $5 \mathrm{ml}$ FACS tube. An antibody cocktail consisting of the antibodies outlined in

298 Supplemental Table 9 was then added to each tube. The samples were vortexed and incubated at 299 RT for 15 mins in the dark. The samples were washed with $500 \mu$ of PBS followed by 300 centrifugation at $800 \times \mathrm{g}$ for 7 minutes at $4^{\circ} \mathrm{C}$. The supernatant was aspirated and discarded. 301 Hoechst 33342 dye $(10 \mathrm{ug} / \mathrm{ml})$ was then added to each sample and incubated for $30 \mathrm{mins}$ at $37^{\circ} \mathrm{C}$ 302 in the dark. Five hundred microliters of PBS were then added to each sample followed by 303 centrifugation at $800 \times \mathrm{g}$ for $7 \mathrm{mins}$ at $4^{\circ} \mathrm{C}$. The supernatant was then aspirated and discarded. 304 Samples were resuspended in $100 \mu \mathrm{l}$ of $1 \mathrm{X}$ Annexin V binding buffer followed by addition of 305 the Annexin V probe. After the samples were incubated for 15 mins, an additional $400 \mu 1$ of 306 binding buffer was added, and the samples were immediately acquired on an LSR-II flow 
cytometer using standardized acquisition template. Voltage was controlled throughout each longitudinal experiment by calibrating the instrument using rainbow calibration particles (Biolegend). After acquisition, data were compensated in FlowJo (Treestar, Inc), and then, uploaded to Cytobank for analysis. Frequencies of erythroid cells out of the erythroid population were exported and used in analyses. Other markers in the panel were either not significantly different or are beyond the scope of this manuscript.

Erythropoietin quantification. EPO was measured as previously described [56] with minor

315 modifications. Frozen plasma isolated from whole blood collected in EDTA was thawed on ice.

316 Each aliquot was then centrifuged at $750 \times g$ for 15 mins at $4^{\circ} \mathrm{C}$. The supernatant was collected

317 and used in the Quantikine IVD ELISA kit from R\&D systems following the manufacturer's

318 suggested protocol except that the amount of suggested sample for the assays was reduced by

$31950 \%$ compared to what is suggested. EPO concentrations were determined using a 5-point

320 standard curve that best fit the dynamic range of where the samples fell on the curve.

322 Computation of the removal of uninfected RBCs. The degree of removal of uninfected RBCs

323 was computed with a discrete recursive mathematical model of the RBC dynamics, as previously

324 performed for data generated from macaques infected with P. cynomolgi or P. coatneyi [57, 58].

325 Briefly, modeling the infection trajectory of each monkey was accomplished by fitting an

326 adaptation of the former model $[58,59]$ to the experimentally determined data. The model has

327 four variables, representing reticulocytes, RBCs, iRBCs and free merozoites. The first three have

328 a lifetime age-structure stratified into one-hour intervals, while the iRBCs are modeled with only

32924 one-hour age-classes, thus enforcing the approximate 24-hour cycle of P. knowlesi. The 
330 model was parameterized with an RBC hazard function determined for rhesus macaques [58]

331 using data from [60]. This hazard function allows the estimation of the level of RBC loss due to

332 senescence. All other model estimates, reticulocyte maturation time, erythropoietic output, RBC

333 loss due to the bystander effect, RBC loss due to parasitization and immune responses against

334 iRBCs, are determined in a "personalized" manner, namely based on experimental data

335 generated from each animal. The full model description can be found elsewhere [58].

337 Statistical analyses. Data were generally divided into two categories for these analyses. Samples

338 that were collected daily, namely parasitaemias, haemoglobin, reticulocyte counts, and platelet

339 counts were divided into 5-day intervals and compared across the course of infection using a

340 linear mixed effects model that accounted for date and monkey drop out due to sacrifice

341 (Supplemental Figs 1-3). Direct comparisons of tissue scores were performed using a Tukey

342 HSD test. Statistics and figures were produced in R Studio version 1.1.383, under R version

343 3.4.3 GUI version 1.70, or JMP Pro version 13.0.0. Associations were tested using the

344 Spearman's correlation test, and hierarchical multiple linear regression analyses.

345 Multicollinearity was assessed using the olsrr package in R. Graphs were prepared using JMP

346 Pro version 15. Comparisons were considered significant when FDR-adjusted, where

347 appropriate, p-values below 0.05 . 


\section{RESULTS}

362 and E35) and brief descriptions of the experimental plans are summarized in Supplemental Table

363 1. Schematics with parasitaemias, as well as sampling and necropsy time points for each animal

364 by cohort are displayed in Supplemental Figures 1-3.

Kra monkeys controlled acute $P$. knowlesi parasitaemia and developed chronic infections

All kra monkeys infected in this study $(n=15)$ developed patent blood-stage infections 6-

3697 days post-inoculation (dpi) with cryopreserved P. knowlesi sporozoites, and they controlled the

370 acute phase parasitaemia without the need for anti-malarial intervention (Figs. 1a, 1b). The

371 average $P$. knowlesi parasite replication rate between patency and peak parasitaemia was 8.59-

372 fold \pm 1.719 (mean \pm SEM) per cycle, which is comparable to the value of 10 -fold, which is

373 accepted in the literature and was determined for rhesus monkey infections with $P$. knowlesi and

374 in vitro culture using rhesus erythrocytes [39, 40, 62]. Most primary parasitaemic peaks averaged

375 about 1,000 parasites/ $\mu$; however, three of the 15 monkeys developed parasitaemias that 
exceeded 40,000 parasites/ $\mu$, which is equivalent to about $1 \%$ iRBCs out of the total RBCs

377 enumerated (Fig. 1a). To identify the time where the kra monkeys began controlling the

378 parasitaemia and when chronic infections began to develop, the mean parasitaemia across five-

379 day increments was calculated for each individual and compared. Parasitaemias peaked 6-15 dpi,

380 declined significantly by 21-25 dpi, and began to stabilize by 31-35 dpi where they persisted at

381 or below 200 parasites/ $\mu$ l (Fig. 1c). Overall, the parasitaemia kinetics in the kra monkeys was

382 similar to those reported in previous studies irrespective of the $P$. knowlesi infections being

383 initiated with sporozoites or blood-stages [35, 39, 40, 47]

\section{Kra monkeys infected with $P$. knowlesi developed mild to moderate thrombocytopaenia}

Platelet levels were stable in the P. knowlesi-infected kra monkeys through $7 \mathrm{dpi}$, and

387 then they began to rapidly decrease through 10 dpi while parasitaemia was rising, even though

388 this decrease was not significantly different (Fig. 2a). After parasitaemias peaked, platelet counts

389 began increasing and returned to baseline levels by 21-25 dpi (Figs. 2a, 2b). The monkeys

390 experienced mild to moderate thrombocytopaenia, as reported previously for $P$. cynomolgi and

$391 P$. coatneyi infection of rhesus monkeys [52, 60, 63], with platelet nadirs as low as 129,000

392 platelets/ $\mu 1$ (Fig. 2a). Importantly, none of the animals developed severe thrombocytopaenia,

393 defined as platelet nadirs below 50,000 platelets/ $\mu$ [ [64]. As platelet levels decreased, the mean

394 platelet volume (MPV) remained high but then significantly declined after about 13 dpi and

395 remained lower than baseline throughout the rest of the infection (Fig. 2c).

397 Moderate to severe anaemia developed during $P$. knowlesi infections in kra monkeys due to 

blood count (CBC) analysis to evaluate the development of anaemia. Haemoglobin levels were stable through 8-10 dpi, but then sharply decreased after the peak of parasitaemia through 20 dpi

402 (Figs. 3a, 3b). Two monkeys developed haemoglobin nadirs as low as $4.8 \mathrm{~g} / \mathrm{dL}$, corresponding to severe anaemia $(<7.0 \mathrm{~g} / \mathrm{dL})$ with about a $55 \%$ loss in haemoglobin relative to baseline (Figs.

$4043 a, 3 b)$. Haemoglobin levels began to increase about 21 dpi and continued to increase throughout 405 the remainder of the infection period (Figs. 3a, 3b). Despite the dramatic loss in haemoglobin, 406 the haemoglobin levels rebounded without anti-malarial or clinical intervention and were on 407 average greater than $11 \mathrm{~g} / \mathrm{dl}$ by 36 dpi until the end of the study (Figs. 3a, 3b). Mild anaemia 408 persisted until the end of the experiment, and the animals never fully recovered to their pre409 infection haemoglobin levels (Figs. 3a, 3b), akin to previous reports with patients [24].

411 anaemia in rhesus monkeys with $P$. coatneyi or $P$. cynomolgi infections $[56,60]$, as well as in 412 humans and rodents infected with malaria parasites [65-68]. Thus, it was hypothesized that 413 inefficient erythropoiesis may contribute, at least in part, to the development of anaemia in $P$. 414 knowlesi infected kra monkeys. Contrary to this hypothesis, however, peripheral reticulocyte 415 counts steadily increased as haemoglobin levels decreased, and they peaked a couple of days 416 after the haemoglobin levels reached their nadirs (Figs. 3c-e). To evaluate if the increase in 417 reticulocyte levels was appropriate to compensate for the decrease in haemoglobin levels, the 418 reticulocyte production index (RPI) was calculated. Indeed, the RPI was sustained over 2 from 419 about 17-26 dpi, indicating that there was adequate compensation by the bone marrow (BM), 420 following the dropping haemoglobin levels, and no evidence of inefficient erythropoiesis was 421 found (Fig. 3e). 
To confirm if the increase in peripheral reticulocyte levels was due to upregulation of

423 erythropoiesis in the BM, the frequency of erythroid lineage cells in the whole BM aspirates

424 collected prior to infection and at 8-11 dpi was evaluated by flow cytometry. The gating strategy

425 used to quantify the frequency of erythroid progenitor cells in the BM aspirates is described in

426 Supplemental Fig. 4. The erythroid lineage cells were defined as CD41a-CD45-LiveDead-

427 CD71+Hoechst+ cells. The frequency of erythroid lineage cells was increased in the BM

428 aspirates, acquired by 10 or $11 \mathrm{dpi}$, compared to baseline (Figs. 3f-g). Consistent with the flow

429 cytometry data, haematoxylin and eosin (H\&E)-stained BM specimens acquired at necropsies as

430 early as 10 dpi revealed substantial expansion of erythroid progenitors relative to uninfected

431 controls (Fig. 3h). Correspondingly, plasma erythropoietin (EPO) levels were significantly

432 elevated above baseline levels at these times for a subset of animals where plasma samples were

433 available (Fig. 3i).

434 Since there was no evidence of inefficient erythropoiesis in the $P$. knowlesi-infected kra

435 monkeys and the relatively low parasitaemia could not account for the observed decrease in

436 haemoglobin levels, the degree of removal of uninfected RBCs was computed using a

437 mathematical model devised for this purpose [58]. Modeling of the kra monkey data showed an

438 appropriate timely compensatory erythropoietic response, with $68.9 \% \pm 4.9 \%$ (mean \pm SEM)

439 attributable to the removal of uninfected bystander RBCs (Fig. 3j). The model estimated that the 440 parasite was responsible for less than $0.5 \%$ of the total RBC losses (Supplemental Fig. 5).

442 Kra monkeys infected with $P$. knowlesi developed fevers that self-resolved

443 Densely sampled temperature data were analyzed from the kra monkeys that had 444 surgically implanted telemetry devices $(n=7$, Supplemental Table 1$)$ to study their temperature 
445 patterns including possible fever after infection (Fig. 4a). Fever was defined as any increase

446 above an individual's baseline range of temperatures, which represent each monkey's daily

447 circadian rhythm. All kra monkeys exhibited a fever by 7 dpi corresponding roughly to the

448 period in which the blood-stage infection reached patency, between 6-8 dpi (Figs. 4a, 4b). The

449 average temperature of the animals significantly increased from pre-infection values of $36.89 \pm$

$4500.17{ }^{\circ} \mathrm{C}$ (mean $\pm \mathrm{SEM}$ ) to $37.87 \pm 0.24^{\circ} \mathrm{C}$ and $38.39 \pm 0.23^{\circ} \mathrm{C}$ by $6-10$ and $11-15$ dpi,

451 respectively (Fig. 4c). The febrile threshold [62] was defined as the parasitaemia at which the kra

452 monkeys developed temperatures and determined to be about $160 \pm 71.58$ parasites/ $\mu$ l (mean \pm

453 SEM). After parasitaemia was controlled, the average temperatures decreased to pre-infection

454 levels of $36.79 \pm 0.29{ }^{\circ} \mathrm{C}$ and $36.48 \pm 0.26{ }^{\circ} \mathrm{C}$ for the day $16-20$ and $21-25$ time periods,

455 respectively (Figs. $4 \mathrm{a}$ and 4c). Monkeys that proceeded to establish chronic low-level

456 parasitaemia no longer experienced temperature spikes (Figs. 4a, 4c).

457

\section{P. knowlesi infection in kra monkeys caused mild to moderate tissue pathology}

An extensive analysis was performed of H\&E-stained tissue sections acquired from up to

$461 \mathrm{dpi}$, to determine if and the extent to which the monkeys may have sustained tissue damage.

462 Hyperplasia, interstitial thickening, and fibrosis were observed in the lungs of nearly all monkeys

463 (13 / 15), and mild to moderate haemorrhage occurred in four of them (Fig. 5a and Supplemental

464 Table 2). All monkeys had Kupffer cell hyperplasia and evidence of liver inflammation based on

465 cellular infiltrates around the periportal region and congestion in the hepatic sinuses (Fig. 5a). No

466 hepatocellular necrosis or cholestasis was noted. The kidneys of all animals showed glomerular

467 hypercellularity without glomerulonephritis, and three animals had tubular degeneration (Fig. 5a 
468 and Supplemental Table 2). One monkey with high parasitaemia and elevated creatinine had

469 evidence of calcification or crystalline deposition in the kidney tubules (Fig. 5a and

470 Supplemental Table 2). Less common kidney pathologies included interstitial inflammation and

471 haemorrhage (Supplemental Table 2). Gastrointestinal inflammation was observed in the tissues

472 from each monkey and included gastritis, duodenitis, jejunitis, and mild colitis without oedema.

473 The spleens from the infected animals were grossly enlarged and weighed significantly more

474 than the spleens from uninfected animals; the day of necropsy did not influence these results

475 (Supplemental Table 3). The adrenal glands, aorta, cerebrum, cerebellum, midbrain, eyes, lymph

476 nodes, omentum, skeletal muscle, testis, and thymus were unremarkable.

477 The severity of the observed tissue damage in each tissue except for the BM and spleen

478 was scored for each animal to facilitate a semi-quantitative comparison of tissue damage

479 between organs (Supplemental Table 2) as performed previously with tissues from P. vivax

480 infected Saimiri boliviensis monkeys [55]. The severity of tissue damage in the lungs and liver

481 was significantly higher than other tissues that sustained pathology (Fig. 5b and Supplemental

482 Table 4). The date of necropsy - which differed for the animals - did not affect the

483 histopathological scores, indicating that how long an animal was parasitaemic did not have a

484 detectable impact on the severity of tissue damage (Supplemental Table 5). In line with the

485 absence of cholestasis and hepatocellular necrosis, aspartate aminotransaminase (AST) and

486 alanine aminotransferase (ALT) remained unchanged during infection, and while bilirubinaemia

487 was observed, it resolved by 11-15 dpi (Fig. 5e). Consistent with kidney pathology, serum

488 creatinine levels were significantly elevated 11-20 dpi before returning to baseline levels (Fig.

489 5f). Notably, creatinine levels varied significantly between individuals after 20 dpi (Fig. 5f). 


\section{Parasite accumulation in the vasculature is associated with severity of tissue damage}

492 To determine if the severity of damage in each tissue was associated with parasite 493 accumulation within that tissue, the number of infected RBCs (iRBCs) in 10 high-power fields 494 (HPFs) was enumerated (Supplemental Table 6). This analysis provided approximate iRBC 495 densities within each tissue, for 22 tissue types, as also performed previously with $P$. vivax 496 infected S. boliviensis tissues [55]. A representative image of parasites identified in tissues is 497 shown in Fig. 6a. Multiple analyses were then used to assess if the accumulation of parasites in 498 the tissues was associated with the tissue damage scores (Supplemental Table 2). First, a 499 univariate analysis was performed between tissue scores and iRBC density. Parasite iRBC 500 burden weakly positively correlated with tissue score $(\rho=0.265$, p-value $=0.00022)$. Next, a 501 hierarchical multiple linear regression analysis was performed to determine which factors 502 affected tissue scores the most (Supplemental Table 7). A model considering only iRBC tissue 503 burden did not result in a linear relationship (adjusted $\mathrm{R}^{2}=0.0354, \mathrm{p}$-value $=0.0054$ ). However, 504 when combined with the tissue type, the relationship was moderately linear (adjusted $\mathrm{R}^{2}=0.678$,

$505 \mathrm{p}$-value $\left.=2.2 \times 10^{-16}\right)$, indicating that the iRBC burden in a tissue may contribute to the degree of 506 damage observed. The day of necropsy, parasitaemia at necropsy, and cumulative parasitaemia 507 were also tested and were found not to contribute to the relationship (Supplemental Table 8). No 508 multicollinearity was noted. 


\section{DISCUSSION}

522 This longitudinal infection study defines clinical, physiological and pathological changes

523 that occurred in kra monkeys after being experimentally infected with $P$. knowlesi sporozoites.

524 Consistent with kra monkeys being a resilient host, they controlled their parasitaemia soon after

525 becoming patent and did not develop severe thrombocytopaenia, a complication that has been

526 observed with severe human Plasmodium infections [reviewed in 64], and most notably with $P$.

527 knowlesi $[1,24,25]$, as well as some $P$. cynomolgi or $P$. coatneyi infections in rhesus monkeys

528 [52, 60]. Recently, thrombocytopaenia has been associated with platelet-Plasmodium iRBC

529 interactions that result in the killing of those infected cells [69]. While platelet killing of $P$.

530 knowlesi-iRBCs was not evaluated in the current study, it is certainly conceivable that platelets

531 could have a killing function in this animal model. In fact, the mild thrombocytopaenia observed

532 here would be fitting with platelets having a role in eliminating parasites and contributing to the

533 lower level of iRBCs in this resilient host. It would be of extreme interest to test this possibility

534 and establish direct comparisons of parasitaemia and thrombocytopaenia with platelet killing

535 assays also performed during rhesus infections, where the parasitaemia escalates unabated to 536 high levels. 
A precipitous and dramatic drop of haemoglobin occurred during the peak of $P$. knowlesi

538 parasitaemia in the kra monkeys and continued until the parasitaemia was controlled. This

539 resulted in haemoglobin nadirs within the documented range of moderate to severe malarial

540 anaemia experienced by $P$. cynomolgi or $P$. coatneyi-infected rhesus macaques $[52,60,63,70]$,

541 humans with Plasmodium parasites [reviewed in 71], and notably children with P. knowlesi [26].

542 These haemoglobin kinetics are reproducible in NHPs infected with Plasmodium spp. and

543 virtually indistinguishable from the haemoglobin kinetic data generated retrospectively from

544 neurosyphilitic patients infected with Plasmodium vivax [72]. The development of severe

545 anaemia in kra monkeys infected with $P$. knowlesi has not been previously reported and these

546 data raise the intriguing point that these animals demonstrated natural resilience to their infection

547 despite demonstrating this disease manifestation. This raises questions regarding what

548 mechanisms are used by these animals to overcome the anaemia.

549 Interestingly, in this regard, in contrast to inefficient erythropoiesis reported for humans,

550 rhesus macaques, and rodents infected with malaria parasites [56, 60, 63, 65-68, 70], there was

551 no evidence in the P. knowlesi-infected kra monkeys that inefficient erythropoiesis contributed to

552 the development of their anaemia. Instead, the kra monkey BM responded appropriately when

553 EPO was elevated in the plasma, comparable to data shown recently for four kra monkeys

554 infected with $P$. coatneyi-infected RBCs and monitored for anaemia [70]. Thus, the

555 mechanism(s) that apparently limits an EPO-dependent response to malarial anaemia in humans,

556 rhesus macaques, and rodent malaria parasite model systems, appears to be absent in kra

557 monkeys, thereby, enabling kra monkeys to sufficiently compensate for the loss of RBCs during

558 P. knowlesi malaria. The data presented here suggest that preserving the BM's ability to

559 compensate for the loss of RBCs is a noteworthy characteristic of resilience displayed by the kra 
560 monkeys to $P$. knowlesi infections. Future comparative studies should explore soluble factors

561 such as inflammatory molecules, metabolites, growth factors, etc., that are different between kra

562 and rhesus monkeys infected with $P$. knowlesi to identify factors that may contribute to

563 inefficient erythropoiesis in the rhesus but not the kra monkey.

564 Previous malarial anaemia studies based upon rhesus macaques infected with $P$.

565 cynomolgi or $P$. coatneyi could not readily distinguish the contribution of inefficient

566 erythropoiesis versus removal of uninfected erythrocytes because these processes occurred

567 sequentially $[52,60,63,70]$. In rhesus macaques infected with $P$. cynomolgi or P. coatneyi, there

568 is apparent inefficient erythropoiesis that occurs while haemoglobin levels begin to drop [52, 60,

$56963,70]$. This is followed by a rapid decline in haemoglobin levels despite a compensatory

570 response by the $\mathrm{BM}[52,56,60,63,70]$. Mathematical modelling of $\mathrm{RBC}$ haemodynamics in

571 rhesus monkeys infected with $P$. coatneyi suggested that removal of uninfected erythrocytes

572 contributed more to anaemia in the $P$. coatneyi infected rhesus monkeys than inefficient

573 erythropoiesis [57]. Significant loss of uninfected RBCs was also predicted based on the

574 retrospective modeling of $P$. falciparum parasitaemia and anaemia data from neurosyphilis

575 patients undergoing malariotherapy [73] and demonstrated in clinical studies involving $P$.

576 falciparum and $P$. vivax [74]. Still, the extent that inefficient erythropoiesis contributed to the

577 exacerbation of anaemia in the animal models, dominated by the removal of uninfected RBCs,

578 remained unclear. Curiously, the kra monkeys in this study displayed no evidence of inefficient

579 erythropoiesis yet maintained similar adverse haemoglobin kinetics as determined from rhesus

580 monkey models [52, 56, 60, 63, 70] and human data [reviewed in 71]. In kra monkeys infected

581 with $P$. coatneyi, the observed erythropoietic response was suboptimal, and alone did not explain

582 the anaemia observed in this species [70]. Overall, with malaria caused by various Plasmodium 
583 species, it appears that the removal of uninfected RBCs may contribute more than inefficient

584 erythropoiesis to the development of malarial anaemia than previously appreciated, as also

585 generally concluded by Jakeman and colleagues [73].

586 Kra monkeys infected with $P$. knowlesi are well-suited to study the possible physiological

587 and immunobiological mechanisms that lead to the removal of uninfected RBCs, whether in

588 malaria-naïve or semi-immune animals [60]. Interestingly, recent RBC deformability studies

589 showed that the ability of uninfected RBCs to become deformable was not affected in $M$.

590 fascicularis $(n=3$, bred and grown in animal facilities at Nafovanny in a malaria-free

591 environment in Vietnam) after being infected for 8 days with P. knowlesi (UM01 strain) blood-

592 stage parasites, and echinocyte formation was not observed [49]. These findings contrast with

593 that group's complementary results from human infections [49], and published findings from

594 infected rhesus monkeys [75, 76], in support of the idea that the absence of these adverse

595 rheologic features and the maintained deformability of RBCs in the kra monkeys supports their

596 resilience. Future studies are warranted that look more closely at all of these factors side-by-side

597 with both monkey species, to hone in on the various mechanisms that may support the resilient

598 phenotype.

Acute Plasmodium infections caused by different parasite species - whether in NHPs or

600 humans - are known to cause damage to tissues and vital organs and contribute to clinical 601 complications and possibly death [reviewed in 32]. Anderios and colleagues described

602 histological changes in the spleen and liver of two kra monkeys (born and bred at the Institute for

603 Medical research in Kuala Lumpur) after 60 or 90 days of infection with blood-stage parasites

604 derived from the blood of a human patient or a cryopreserved isolate from an infected rhesus

605 (obtained from the American Type Culture Collection (ATCC)) [48]. The current longitudinal 
study with 15 kra monkeys is much more comprehensive with the histopathological analysis of

60722 tissues. Here, histological changes in the liver, lungs, kidneys, and the gastrointestinal tract

608 were identified, but consistent with kra monkeys being a resilient host, the tissue lesions

609 observed were relatively mild (Supplementary Table 2). These results can be contrasted with the

610 severe tissue damage previously reported from $P$. knowlesi, P. coatneyi or P. cynomolgi

611 infections in rhesus macaques [45, 77-80].

Consistent with the mild histological changes observed in this study of kra monkeys,

613 there were only a few indications of organ dysfunction. Nearly all animals had some degree of

614 tissue damage in the lungs, and the lungs had the highest iRBC count per high power field.

615 Creatinine levels became elevated at the peak of parasitaemia and remained elevated, indicative

616 of kidney issues. Given the minor tissue-damage observed in the kidneys, it is possible that the

617 increased creatinine could be related to an increase in antigen-antibody immune complex

618 deposition in the kidney tubules or a decrease in fluid intake. Regardless, it is relevant to note

619 that increased creatinine has been a common finding in patients with severe malaria [29].

620 Hyperbilirubinaemia has also been associated with severe P. knowlesi malaria in humans [24].

621 Likewise, bilirubin was elevated in the kra monkeys. Although direct and indirect bilirubin levels

622 were unavailable, the absence of significant histological evidence for hepatic injury such as

623 cholestasis suggests that the elevated total bilirubin levels in the kra monkeys were due to

624 hemolysis of uninfected RBCs. In agreement with this hypothesis, bilirubinaemia returned to

625 baseline in the latter part of the infections, coincident with the recovery of haemoglobin levels.

626 Neither ALT nor AST were elevated at any stage in the infections, which is consistent with the

627 absence of hepatocyte necrosis, or signs of major tissue damage systemically. Together, these

628 data suggest that kra monkey resilience to $P$. knowlesi infection, as demonstrated by their control 
629 of parasitaemia and compensation for anaemia, is reflected by only minimal or mild tissue 630 damage and organ dysfunction.

631 The presence of iRBCs in various tissues have been reported from previous studies with

632 P. knowlesi infected rhesus macaques [44, 45]. Likewise, P. knowlesi iRBCs were identified in

633 the tissues of the kra monkeys in this study and they predominated in the spleen, adrenal glands,

634 lungs, kidneys, and gastrointestinal tract. However, relatively few iRBCs were identified, which 635 is consistent with the comparatively low number of circulating parasites in the resilient kra

636 monkey species. Regardless, similar as determined in prior studies with $P$. vivax infection of

637 Saimiri boliviensis monkeys [55], there was a modest relationship in the current study between

638 histopathology score and parasite accumulation in the tissues.

639 Understanding the immune responses and pathophysiological processes that occur during

$640 P$. knowlesi infections in humans has been of great interest but studying these infections

641 longitudinally in humans is not feasible due to ethical requirements to treat individuals once they

642 are parasitaemic. Plasmodium knowlesi-infected kra monkeys are a viable alternative for

643 studying the progression of acute and chronic infections, and without drug treatment being a

644 confounding factor. Unlike infections of rhesus monkeys with various Plasmodium species, the

645 P. knowlesi-infected kra monkeys uniformly develop naturally occurring chronic infections [39,

$64640,46,47]$, and with similarly low parasitaemia levels as recorded for uncomplicated P. knowlesi

647 infections in humans [24]. Importantly, while the kra monkeys demonstrated resilience from

648 severe and deadly disease, chronicity was characterized similar to human infections by persistent

649 and lower than normal haemoglobin levels. These results warrant further exploration in the 650 context of this animal model to understand the loss of the uninfected RBCs and to determine 651 what host responses may be occurring. 
$653 P$. falciparum infections [25]. Given recent findings that macaque RBC culture-adapted $P$.

654 knowlesi parasites could be adapted to human RBCs by acquisition of sialic acid-independent

655 invasion mechanisms, one hypothesis to explain this is that $P$. knowlesi is not as well-adapted to

656 humans, and, thus, can trigger immune responses more quickly than P. falciparum [81].

657 Therefore, it was expected that kra monkeys, as a natural host of $P$. knowlesi, would develop

658 fevers once high parasitaemias developed. However, the kra monkeys developed fevers shortly

659 after their infections reached patency, and their pyrogenic thresholds were about 160

660 parasites $/ \mu 1$. These data suggest that the lower pyrogenic threshold observed for $P$. knowlesi in

661 humans may not solely be due to poor adaptation to humans. Kra monkeys may have evolved

662 adaptations in pattern recognition receptors that enable them to detect parasite-derived molecules

663 at lower levels than other primates, thereby, enabling the immune system to detect and respond

664 to P. knowlesi more quickly, as shown in one study with a few animals [46].

As this research field advances, delving deeper into specific host-parasite interactions and

666 pathways, it is important to take into consideration the origin and genetic background of the host

667 monkeys and the infecting parasites, as well as prior experimental history of the animals and

668 whether experiments are initiated with sporozoites or blood-stage parasites. The source and

669 validation of the monkey and parasite species are critical. Thus, we have taken care to indicate

670 the macaque monkey and $P$. knowlesi parasite sources and isolate information in this manuscript,

671 and to the extent available for referenced papers [48, 49]. As early as 1932, major differences

672 and nuances of $P$. knowlesi parasite-host combinations were described by Napier and Campbell

673 [82] and Knowles and Das Gupta [38], and such data summarized then from numerous NHP

674 infections, and since by others [39, 40, 83, 84], including instances of 'hybrid' short-tailed 
$675 \mathrm{kra} /$ cynomolgus-rhesus monkeys [83], may bring important if not critical insights and value to 676 current research.

This study reports a systematic analysis of $P$. knowlesi-infected kra monkeys to identify basic features of resilience to malaria, which could be relevant for infections caused by multiple

687 species of Plasmodium. This investigation affirms previous reports that kra monkeys are

688 resilient to $P$. knowlesi infections and shows that their resilience is not - at least in part - because

689 they completely subvert development of clinical signs of malaria and avoid tissue-damage.

690 Instead, while kra monkeys prevent P. knowlesi parasitaemia from rising above 1-3\% they mount

691 compensatory physiological responses that may reverse disease progression and limit tissue

692 pathology. The kra monkey-Plasmodium infection model system is valuable for studying

693 mechanisms of resilience to malaria and identifying specific physiological and immunobiological

694 responses that may function to minimize disease and death in people infected with Plasmodium.

695 Of note, the kra monkey data best reflected the clinical picture of children rather than adults

696 infected with P. knowlesi, as determined in a few studies [26-28]. Continued validation of this

697 animal model is warranted as a complement to human studies aimed at understanding 
698 mechanisms of resilience to malaria, with the possible goal of new host-directed therapies to

699 resolve acute disease states and the progression of chronic Plasmodium infections. Such inquiry

700 has begun, specifically, for example, involving in-depth analysis of the peripheral blood

701 transcriptomes of rhesus and kra monkeys in response to P. knowlesi (Gupta et al., submitted).

702

703

704

705

706

707 FIGURE LEGENDS

708 Fig. 1. Plasmodium knowlesi-infected kra monkeys control parasitaemia without antimalarial

709 treatment and develop persisting parasitaemias. a. Afternoon peripheral parasitaemias

710 determined by thick and/or thin blood films for $15 \mathrm{kra}$ monkeys infected with about 100,000

711 cryopreserved P. knowlesi H strain sporozoites. Dashed line indicates approximately 40,000

712 parasites $/ \mu 1$ or about $1 \%$ parasitaemia. b. Time to patency analysis for monkeys shown in Panel

713 a. c. Average parasitaemia for each animal during the indicated time period after infection. Light

714 gray bars indicate mean of the shown datapoints. Statistical significance was assessed using a

715 linear mixed-effect model followed by a Tukey-Kramer HSD post-hoc analysis. $p$-values of $716<0.05$ were considered statistically significant. Asterisks indicate statistical significance.

718 Fig. 2. P. knowlesi infections in kra monkeys cause mild to moderate thrombocytopaenia. a.

719 Platelet concentration kinetics in relation to parasitaemia before and after infection. Each dot 720 represents the mean for all animals present during infection at that time. b. Average platelet 
721 concentration for each animal during the indicated time period after infection. Light gray bars

722 indicate mean of the shown datapoints. c. Kinetics of platelet concentration in relation to platelet

723 size (MPV) after infection. Statistical significance was assessed using a linear mixed-effect

724 model followed by a Tukey-Kramer HSD post-hoc analysis. $p$-values of <0.05 were considered

725 statistically significant. Error bars = SEM. Asterisks indicate statistical significance.

726

727 Fig. 3. Kra monkeys experience moderate to severe anaemia after infection with $P$. knowlesi that

728 is attributed to $\mathrm{RBC}$ removal rather than inefficient erythropoiesis. a. Haemoglobin concentration

729 kinetics in relation to parasitaemia during infection. Each dot represents the mean for all animals

730 where data were available at that time during infection. b. Average haemoglobin concentration

731 for each animal during the indicated time period after infection. Light gray bars indicate mean of

732 the shown datapoints. c. Platelet concentration kinetics in relation to parasitaemia before and

733 after infection. Each dot represents the mean for all animals where data was available at that time

734 during infection. d. Average reticulocyte concentration in peripheral blood for each animal

735 during the indicated time period after infection. Light gray bars indicate mean of the shown

736 datapoints. e. Relationship of haemoglobin and reticulocyte concentration kinetics with

737 reticulocyte production index during infection. Each dot represents the mean for all animals

738 where data were available at that time during infection. f. Representative flow cytometry plots

739 showing the relative quantification of erythroid progenitors present in bone marrow aspirates

740 collected before and during infection. Percentages in blue indicate the percentage of each

741 population in the gate out of the erythroid compartment defined as SscloCD41a-CD45-

742 LiveDead-. Gating strategy can be found in Supplementary Fig. 4. g. Relative quantification of

743 erythroid progenitors as in Panel f. h. Representative H\&E-stained bone marrow samples from 
744 uninfected, malaria and $P$. knowlesi infected kra monkeys at the indicated time points. i.

745 Quantification of erythropoietin by ELISA in the plasma of a subset of kra monkeys after

746 infections with P. knowlesi. j. Quantification of processes resulting in the elimination of RBCs

747 using a recursive mathematical model. Statistical significance was assessed using a linear mixed-

748 effect model followed by a Tukey-Kramer HSD post-hoc analysis. $p$-values of $<0.05$ were

749 considered statistically significant. Error bars = SEM. Asterisks indicate statistical significance.

751 Fig. 4. Kra monkeys develop fevers shortly after patency, which resolve after the control of

752 parasitaemia. a. Temperature kinetics in relation to parasitaemia in animals that had telemetry

753 devices surgically implanted. b. Time to fever analysis using the telemetry data in panel a. c.

754 Average temperature for each animal during the indicated time period after infection. Light gray

755 bars indicate mean of the shown datapoints. Error bars = SEM. Bar graphs and error bars

756 represent mean \pm SEM. Statistical significance was assessed using a linear mixed-effect model

757 followed by a Tukey-Kramer HSD post-hoc analysis. $p$-values of $<0.05$ were considered

758 statistically significant. Asterisks indicate statistical significance.

759

760 Fig. 5. Plasmodium knowlesi-infected kra monkeys exhibit histopathology consistent with

761 systemic measures of disease. a. Representative light micrographs of H\&E-stained tissue

762 sections from control and infected kra monkeys. Infected lungs showed areas of fibrosis (Fib),

763 hyperplasia (*), haemolysis (Hem), and interstitial thickening (IT). All animals had periportal (*)

764 and sinusoidal (SI) infiltration in the liver. Kidney histopathology included tubular crystal

765 formation (TC), hypercellular glomeruli (*), and tubular degeneration (TD). The stomach,

766 duodenum, jejunum, and colon all exhibited mucosal immune infiltration (*). b. Semi- 
767 quantitative histopathology scores of tissues collected at necropsy. The average alanine

768 transaminase (c), aspartate aminotransferase (d), total bilirubin (e), and creatinine (f) for each

769 animal where data were available during the indicated time period after infection. Light gray bars

770 indicate mean of the shown datapoints. Error bars = SEM. Statistical significance was assessed

771 using a linear mixed-effect model followed by a Tukey-Kramer HSD post-hoc analysis. $p$-values

772 of $<0.05$ were considered statistically significant. Asterisks indicate statistical significance.

773

774 Fig. 6. Parasites accumulate in the tissues of $P$. knowlesi-infected kra monkeys. a. Representative

775 micrograph showing parasites (arrows) in H\&E-stained gastrointestinal submucosa. b. Average

776 parasite counts in 10 high power fields of 22 different tissues from $P$. knowlesi-infected kra

777 monkeys.

778

779

780

781

782

783

784

785

786

787

788

789 


\section{SUPPLEMENTAL TABLE LEGENDS}

800 Supplemental Table 1. Macaque Cohort and Experimental Summaries. Details regarding four 801 monkey cohorts involved in the current study are summarized; three of these were 802 experimentally infected with $P$. knowlesi sporozoites, and one served as control group. The 803 cohorts are listed in the order in which experiments using these animals were performed. These 804 animals and their longitudinal infection designs were part of a systems biology program, with

805 iterative cohort experimentation designed to satisfy the goals of those research programs. The 806 non-sequential experimental numbering (E07, E33, E34, and E35) reflects the experimental 807 numbers assigned in the MaHPIC Laboratory Information Management System. Telemetry data 808 were collected for temperature, blood pressure, heart rate and activity level (manuscript in 809 preparation). Spx is an abbreviation for splenectomy.

811 Supplemental Table 2. Summary of histopathology scores for P. knowlesi-infected

812 macaques. Semi-quantitative scores are presented as a heatmap to visually represent severity, 813 with 0 being no changes or damage, and 4 being diffuse changes. Organs were scored in several 
814 categories, including inflammation, oedema, crypt inflammation (for the gastrointestinal organs),

815 necrosis, haemorrhage, hyperplasia (including alveolar wall thickening/interstitial hyperplasia,

816 for the lungs, Kupffer cell hyperplasia in the liver, and glomerular hyperplasia for the kidneys),

817 fibrosis, vasculitis, and tubular degeneration (for kidneys). The histopathology categories shown

818 reflect the range of pathologies noted, whether for one or multiple monkeys.

820 Supplemental Table 3. Linear regression: spleen weight $v s$. time of infection. The effect of time

821 at necropsy on spleen weight was tested via a linear regression model and found not to

822 contribute.

823

824 Supplemental Table 4. Tukey HSD Post-hoc pairwise comparison of tissue score.

825 Semiquantitative tissue scores were compared pairwise using Tukey HSD post-hoc analysis.

826 Mean difference is the difference in means between the organ pair being compared. Adjusted $p$ -

827 value, significance and upper and lower bound are included. $* p<0.05 ; * * p<0.005, * * * p$

$828<0.0005, * * * * p<0.00005 ; \mathrm{NS}=$ not significant.

829

830 Supplemental Table 5. Linear regression: pathology score $v s$. necropsy day. The effect of time

831 at necropsy on pathology score was tested via a linear regression model and found not to

832 contribute.

833

834 Supplemental Table 6. Summary statistics for parasite tissue counts. Parasites in H\&E-stained

835 tissue sections for each organ were quantified by light microscopy. Mean number of parasites in 
10 high power fields per H\&E-stained tissue, standard deviation, standard error, and number of

837 sections are presented.

838

839 Supplemental Table 7. Hierarchical Linear Regression Analysis. Multiple Linear Regression

840 (MLR) was performed to test the relationship between score, count, and organ. For organ,

841 adrenal gland was selected as the reference tissue. Parameter is significant at $\alpha=0.05 ; * p<0.05$,

$842 * * p<0.005, * * * p<0.0005$; $\mathrm{NS}=$ not significant.

843

844 Supplemental Table 8. Hierarchical Linear Regression Analysis of Infection Parameters.

845 Multiple Linear Regression (MLR) was performed to test the relationship between score, count,

846 days post-inoculation (DPI), parasitaemia, and cumulative parasitaemia. Parameter is significant

847 at $\alpha=0.05 ; * p<0.05, * * p<0.005, * * * p<0.0005$; NS= not significant.

848

849 Supplemental Table 9. Flow cytometry staining cocktail for measuring erythroid progenitors in

850 rhesus and kra monkey bone marrow aspirates.

851

852

853

SUPPLEMENTAL FIGURE LEGENDS

854

855 Supplemental Fig. 1. E07 Experimental Design and Parasitaemia. Pilot $P$. knowlesi infection in 8567 kra monkeys (11C131, 11C166, 12C36, 12C44, 12C53, H12C59, H12C8) with staggered

857 necropsy endpoints. Top Panel: Schematic of the planned (generalized) experimental design with 858 pre-infection surgical implantation of a telemetry device (scissors) plus recovery time and their 859 activation for collection of physiological data, before (grey bar) and after surgery baseline 
860 timepoint (TP) sample collections (gold bars), P. knowlesi cryopreserved sporozoite inoculations

861 at day 0 (syringe), predicted parasitaemia kinetics (pink curved line) with early infection, log-

862 phase, peaking parasitaemia, and sequential chronic phase TPs indicated for blood and bone

863 marrow sample collections. Necropsy endpoints $\left(^{*}\right)$ were planned for selected animals at the

864 acute and both early and late chronic stages of infection. Bottom Panel: Schematic showing E07

865 experimental data including $P$. knowlesi cryopreserved sporozoite inoculations on day 0

866 (syringe ${ }^{\dagger}$ ), daily parasitaemias graphed (pink line), and defined TPs (gold bars) and the specific

867 days of euthanasia and necropsy endpoints (*) are indicated for each of the animals.

868 No subcurative treatments were required, as the blood-stage infections and clinical signs

869 naturally began to resolve, as expected with kra monkeys. This cohort had previously been

870 inoculated with sporozoites freshly isolated from mosquito salivary glands, yet for reasons

871 unknown, blood-stage parasitaemia did not result. To what extent the immune system may have

872 been stimulated at the time, or not, remains an open question.

873 This pilot experiment allowed for 1) testing of cryopreserved stocks

874 of $P$. knowlesi sporozoites and the kinetics of the parasitaemia in kra monkeys (pink line); 2)

875 capturing blood, bone marrow and tissue data on both the acute and chronic phases of the

876 infection; 3) testing of experimental and analysis pipelines for the generation and analysis of

877 biological data at specific TPs, including the secure and reliable transfer of samples or data as

878 required across the large MaHPIC and HAMMER consortia [85]; and collection and analysis of

879 infected tissue samples from necropsies.

880

881 Supplemental Fig. 2. E33 Experimental Design and Parasitaemia

882 (Iterative $P$. knowlesi infections in a cohort of $4 \mathrm{kra}(13 \mathrm{C} 90,14 \mathrm{C} 15,14 \mathrm{C} 3, \mathrm{H} 13 \mathrm{C} 110)$ 
883 monkeys to study acute infections and also establish and study chronic infections. Top Panel:

884 Schematic of the planned (generalized) experimental design with timepoints (TP) of sample

885 collection (gold bars), P. knowlesi cryopreserved sporozoite inoculations at day 0 (syringe),

886 predicted parasitaemia kinetics (pink line) with early infection, log-phases, peaking parasitaemia,

887 and later TPs indicated for blood and bone marrow sample collections. Necropsy endpoints $(*)$

888 were planned for each species after day 14, prior to the natural decline in parasitaemia as 889 observed for kra monkeys in E07 (Supplemental Fig. 1). Bottom Panel: Schematic showing E33

890 experimental data including $P$. knowlesi cryopreserved sporozoite inoculations on day 0

891 (syringe), daily parasitaemias graphed (pink lines), and defined TPs (gold bars) and the specific

892 days of euthanasia and necropsy endpoints $\left(^{*}\right)$ are indicated for each of the animals. No

893 treatment was required.

895 Supplemental $\quad$ Fig. $\quad 3 . \quad$ E35 $\quad$ Experimental $\quad$ Design $\quad$ and

896 Parasitaemia. Iterative P. knowlesi infection in a cohort of 4 kra monkeys (13C33, 13C74,

897 H13C101, H14C17) to study acute and chronic infections. Top Panel: Schematic of the planned

898 (generalized) experimental design with timepoint (TP) sample collections (gold

899 bars), P. knowlesi cryopreserved sporozoite inoculations at day 0 (syringe), predicted

900 parasitaemia kinetics (pink line) with early infection, log-phases, peaking parasitaemia, and later

901 TPs indicated for blood and bone marrow sample collections. Necropsy endpoints (*) were

902 planned for each species after day 14, prior to the natural decline in parasitaemia as observed

903 for kra monkeys in E07 (Supplemental Fig. 1), and when parasitaemia was still undetectable.

904 Bottom Panel: Schematic showing E35 experimental data including P. knowlesi cryopreserved

905 sporozoite inoculations on day 0 (syringe), daily parasitaemias graphed (pink lines), and defined 
TPs (gold bars) and the specific days of euthanasia and necropsy endpoints (*) are indicated for

907 each of the animals. No treatment was required for the kra monkeys.

909 Supplemental Fig. 4. Flow Cytometry Gating Strategy. Representative flow cytometry gating

910 strategy for measuring erythroid lineage cells in bone marrow aspirate collected from rhesus

911 and kra monkeys.

912

913 Supplemental Fig. 5. Characterization of the dynamics of RBC removal and production

914 processes in a representative kra monkey (12C44) during a $P$. knowlesi infection. To quantify the

915 haemodynamic processes during a $P$. knowlesi infection, a computational dynamical model was

916 used that was previously developed to faithfully track the blood dynamics in Plasmodium

917 infected monkeys [57-59]. The model was formulated as a set of discrete recursive equations,

918 where the pools of reticulocytes, RBCs, and iRBCs were stratified into age classes. The model

919 directly represents that reticulocytes are released from the bone marrow with a certain age and

920 rate, circulate for a day and then mature into RBCs. Pertinent model results (lines) are

921 superimposed on experimental data (symbols). Shown are the circulating reticulocytes (A),

922 mature RBCs (C), and infected RBCs (F), from which the model allowed the quantification of

923 different causes of RBC removal (D and E). The NHP RBCs normally die after about 100 days

924 due to senescence, or on a daily basis due to "random" effects, such as shear stresses (E). During

925 Plasmodium infections, some of the healthy RBCs are also infected by merozoites and destroyed

926 when the parasites are released (parasitization, D) or lost to a bystander effect (D). Interestingly,

927 large numbers of RBCs were lost during the infection due to the bystander mechanism (D). The

928 profile of RBCs (C) demonstrates that the kra monkey had severe anaemia and responded 
929 appropriately by increasing the erythropoietic output and releasing younger reticulocytes,

930 thereby increasing the reticulocyte maturation time in circulation (B).

931

932

933

934

935

936

937

938

939

940

941 DECLARATIONS

942 ETHICS APPROVAL AND CONSENT TO PARTICIPATE

943 All experimental, surgical, and necropsy procedures were approved by Emory's IACUC and the

944 Animal Care and Use Review Office (ACURO) of the US Department of Defense and followed

945 accordingly. The Emory's IACUC approval number was PROTO201700484 - YER-2003344-

946 ENTRPR-A.

947

948 CONSENT FOR PUBLICATION

949 Not applicable

950

951 AVAILBILITY OF DATA AND MATERIALS 
952 All clinical and telemetry data were rigorously validated and quality controlled. The majority of

953 data analyzed here, protocols and extensive metadata are publicly available in PlasmoDB [61]:

954 http://plasmodb.org/plasmo/mahpic.jsp

955 http://plasmodb.org/common/downloads/MaHPIC/Experiment 07/

956 http://plasmodb.org/common/downloads/MaHPIC/Experiment_33/

957 http://plasmodb.org/common/downloads/MaHPIC/Experiment_35/

958 Data not available through public data repositories are included as supplementary files (e.g.,

959 tissue parasite counts are included in S1 Spreadsheet). Data not in public databases or 960 supplementary files (e.g., flow cytometry data files) can be requested directly from the 961 corresponding author.

COMPETING INTERESTS

964 The authors declare that they have no competing interests.

965

966 FUNDING

967 This project was funded in part by the National Institute of Allergy and Infectious Diseases; National

968 Institutes of Health, Department of Health and Human Services, which established the MaHPIC [Contract

969 No. HHSN272201200031C; MRG], the NIH Office of Research Infrastructure Programs/OD

970 P51OD011132, the Defense Advanced Research Program Agency and the US Army Research Office via

971 a cooperative agreement [Contract No. W911NF16C0008; MRG], which funded the Technologies for

972 Host Resilience - Host Acute Models of Malaria to study Experimental Resilience (THoR's HAMMER)

973 consortium.

974

975 AUTHOR CONTRIBUTIONS 
976 Conceived and designed the experiments: MSP, CJJ, JAB, JSW, LLF, RT, JCK, AM, SG, EOV,

977 JBG, RJC, MRG, and members of the MaHPIC-Consortium. Performed the experiments: MSP,

978 CJJ, JAB, JSW, MCM, CLS, LLF, WTC, JJ, SAL, SRS, AH, DM, EK. Performed data analysis:

979 MSP, CJJ, JAB, LLF, SG, JBG. Interpreted the data analysis: MSP, CJJ, RJC, MRG and

980 members of the MaHPIC-Consortium. Managed and led validation and quality control of

981 datasets for clinical and telemetry results and deposited the data and metadata: MVN, JH, JDD,

982 JCK. Generated the figures: MSP, CJJ, LLF, SG. Wrote the paper: MSP, CCJ, MRG. Provided

983 manuscript editorial contributions: LLF, AM, SG, EOV, RJC. All authors reviewed and

984 approved submission of this manuscript.

985

986 ACKNOWLEDGEMENTS

987 The authors thank John W. Barnwell for discussions, bringing knowledge of $P$. knowlesi infections

988 and malaria to this project and the provision of cryopreserved sporozoites. Elizabeth Strobert is 989 thanked for consultations and advice on animal protocols. E-van Dessasau is thanked for 990 technical assistance in preparing and staining histopathological slides. The YNPRC staff are 991 acknowledged for assistance with procedures involving NHPs, and the Emory Pediatric/Winship 992 Flow Cytometry and Yerkes Flow Cytometry Cores are recognized for maintaining core facilities 993 made available in this project.

995 MaHPIC Consortium Members. MaHPIC members participating in discussions at the time of the 996 planning, implementation, or analysis of this project include: Dave C. Anderson, Ferhat Ay, 997 Cristiana F. A. Brito, John W. Barnwell, Megan DeBarry, Steven E. Bosinger, Jung-Ting Chien, 998 Jinho Choi, Anuj Gupta, Chris Ibegbu, Xuntian Jiang, Dean P. Jones, Nicolas Lackman, Tracey 

Zerotti L. Woods.

1005 Not Applicable

\section{REFERENCES}

1. Singh B, Daneshvar C: Human infections and detection of Plasmodium knowlesi. Clin Microbiol Rev 2013, 26:165-184.

2. Barber BE, Rajahram GS, Grigg MJ, William T, Anstey NM: World Malaria Report: Time to acknowledge Plasmodium knowlesi malaria. Malar J 2017, 16:135.

3. Davidson G, Chua TH, Cook A, Speldewinde P, Weinstein P: Defining the ecological and evolutionary drivers of Plasmodium knowlesi transmission within a multi-scale framework. Malar J 2019, 18:66.

4. Singh B, Kim Sung L, Matusop A, Radhakrishnan A, Shamsul SS, Cox-Singh J, Thomas A, Conway DJ: A large focus of naturally acquired Plasmodium knowlesi infections in human beings. Lancet 2004, 363:1017-1024.

5. Cox-Singh J, Singh B: Knowlesi malaria: newly emergent and of public health importance? Trends Parasitol 2008, 24:406-410.

6. Cox-Singh J, Davis TM, Lee KS, Shamsul SS, Matusop A, Ratnam S, Rahman HA, Conway DJ, Singh B: Plasmodium knowlesi malaria in humans is widely distributed and potentially life threatening. Clin Infect Dis 2008, 46:165-171.

1036

7. Cooper DJ, Rajahram GS, William T, Jelip J, Mohammad R, Benedict J, Alaza DA, Malacova E, Yeo TW, Grigg MJ, et al: Plasmodium knowlesi Malaria in Sabah, 
Malaysia, 2015-2017: Ongoing Increase in Incidence Despite Near-elimination of the Human-only Plasmodium Species. Clin Infect Dis 2020, 70:361-367.

8. Divis PCS, Hu TH, Kadir KA, Mohammad DSA, Hii KC, Daneshvar C, Conway DJ, Singh B: Efficient Surveillance of Plasmodium knowlesi Genetic Subpopulations, Malaysian Borneo, 2000-2018. Emerg Infect Dis 2020, 26:1392-1398.

9. Chin AZ, Maluda MCM, Jelip J, Jeffree MSB, Culleton R, Ahmed K: Malaria elimination in Malaysia and the rising threat of Plasmodium knowlesi. J Physiol Anthropol 2020, 39:36.

10. Manin BO, Ferguson HM, Vythilingam I, Fornace K, William T, Torr SJ, Drakeley C, Chua TH: Investigating the Contribution of Peri-domestic Transmission to Risk of Zoonotic Malaria Infection in Humans. PLoS Negl Trop Dis 2016, 10:e0005064.

11. Fornace KM, Abidin TR, Alexander N, Brock P, Grigg MJ, Murphy A, William T, Menon J, Drakeley CJ, Cox J: Association between Landscape Factors and Spatial Patterns of Plasmodium knowlesi Infections in Sabah, Malaysia. Emerg Infect Dis 2016, 22:201-208.

12. Fornace KM, Nuin NA, Betson M, Grigg MJ, William T, Anstey NM, Yeo TW, Cox J, Ying LT, Drakeley CJ: Asymptomatic and Submicroscopic Carriage of Plasmodium knowlesi Malaria in Household and Community Members of Clinical Cases in Sabah, Malaysia. J Infect Dis 2016, 213:784-787.

13. Barber BE, William T, Dhararaj P, Anderios F, Grigg MJ, Yeo TW, Anstey NM: Epidemiology of Plasmodium knowlesi malaria in north-east Sabah, Malaysia: family clusters and wide age distribution. Malar J 2012, 11:401.

14. Vythilingam I, Noorazian YM, Huat TC, Jiram AI, Yusri YM, Azahari AH, Norparina I, Noorrain A, Lokmanhakim S: Plasmodium knowlesi in humans, macaques and mosquitoes in peninsular Malaysia. Parasit Vectors 2008, 1:26.

15. Divis PC, Singh B, Anderios F, Hisam S, Matusop A, Kocken CH, Assefa SA, Duffy CW, Conway DJ: Admixture in Humans of Two Divergent Plasmodium knowlesi Populations Associated with Different Macaque Host Species. PLoS Pathog 2015, 11:e1004888.

16. Fungfuang W, Udom C, Tongthainan D, Kadir KA, Singh B: Malaria parasites in macaques in Thailand: stump-tailed macaques (Macaca arctoides) are new natural hosts for Plasmodium knowlesi, Plasmodium inui, Plasmodium coatneyi and Plasmodium fieldi. Malar J 2020, 19:350.

17. Lee KS, Divis PC, Zakaria SK, Matusop A, Julin RA, Conway DJ, Cox-Singh J, Singh B: Plasmodium knowlesi: reservoir hosts and tracking the emergence in humans and macaques. PLoS Pathog 2011, 7:e1002015.

18. Ta TH, Hisam S, Lanza M, Jiram AI, Ismail N, Rubio JM: First case of a naturally acquired human infection with Plasmodium cynomolgi. Malar J 2014, 13:68.

19. Imwong M, Madmanee W, Suwannasin K, Kunasol C, Peto TJ, Tripura R, von Seidlein L, Nguon C, Davoeung C, Day NPJ, et al: Asymptomatic natural human infections with the simian malaria parasites Plasmodium cynomolgi and Plasmodium knowlesi. J Infect Dis 2018, 219:695-702.

20. Singh B, Kadir KA, Hu TH, Raja TN, Mohamad DS, Lin LW, Hii KC: Naturally acquired human infections with the simian malaria parasite, Plasmodium cynomolgi, in Sarawak, Malaysian Borneo. International Journal of Infectious Diseases 2018, 73:68. 
1083 21. Raja TN, Hu TH, Kadir KA, Mohamad DSA, Rosli N, Wong LL, Hii KC, Simon Divis

1084

1085

1086

1087

1088

1089

1090

1091

1092

1093

1094

1095

1096

1097

1098

1099

1100

1101

1102

1103

1104

1105

1106

1107

1108

1109

1110

1111

1112

1113

1114

1115

1116

1117

1118

1119

1120

1121

1122

1123

1124

1125

1126

1127

1128

PC, Singh B: Naturally Acquired Human Plasmodium cynomolgi and P. knowlesi Infections, Malaysian Borneo. Emerg Infect Dis 2020, 26:1801-1809.

22. Chin W, Contacos PG, Coatney GR, Kimball HR: A Naturally Acquited QuotidianType Malaria in Man Transferable to Monkeys. Science 1965, 149:865.

23. Cox-Singh J, Hiu J, Lucas SB, Divis PC, Zulkarnaen M, Chandran P, Wong KT, Adem P, Zaki SR, Singh B, Krishna S: Severe malaria - a case of fatal Plasmodium knowlesi infection with post-mortem findings: a case report. Malar $J$ 2010, 9:10.

24. Daneshvar C, Davis TM, Cox-Singh J, Rafa'ee MZ, Zakaria SK, Divis PC, Singh B: Clinical and laboratory features of human Plasmodium knowlesi infection. Clin Infect Dis 2009, 49:852-860.

25. Barber BE, William T, Grigg MJ, Menon J, Auburn S, Marfurt J, Anstey NM, Yeo TW: A prospective comparative study of knowlesi, falciparum, and vivax malaria in Sabah, Malaysia: high proportion with severe disease from Plasmodium knowlesi and Plasmodium vivax but no mortality with early referral and artesunate therapy. Clin Infect Dis 2013, 56:383-397.

26. Barber BE, William T, Jikal M, Jilip J, Dhararaj P, Menon J, Yeo TW, Anstey NM: Plasmodium knowlesi malaria in children. Emerg Infect Dis 2011, 17:814-820.

27. Grigg MJ, William T, Barber BE, Rajahram GS, Menon J, Schimann E, Piera K, Wilkes CS, Patel K, Chandna A, et al: Age-Related Clinical Spectrum of Plasmodium knowlesi Malaria and Predictors of Severity. Clin Infect Dis 2018, 67:350-359.

28. Rajahram GS, Barber BE, William T, Grigg MJ, Menon J, Yeo TW, Anstey NM: Falling Plasmodium knowlesi Malaria Death Rate among Adults despite Rising Incidence, Sabah, Malaysia, 2010-2014. Emerg Infect Dis 2016, 22:41-48.

29. Rajahram GS, Cooper DJ, William T, Grigg MJ, Anstey NM, Barber BE: Deaths From Plasmodium knowlesi Malaria: Case Series and Systematic Review. Clin Infect Dis 2019, 69:1703-1711.

30. William T, Menon J, Rajahram G, Chan L, Ma G, Donaldson S, Khoo S, Frederick C, Jelip J, Anstey NM, Yeo TW: Severe Plasmodium knowlesi malaria in a tertiary care hospital, Sabah, Malaysia. Emerg Infect Dis 2011, 17:1248-1255.

31. Daneshvar C, William T, Davis TME: Clinical features and management of Plasmodium knowlesi infections in humans. Parasitology 2018, 145:18-31.

32. World Health Organization: Severe malaria. Trop Med Int Health 2014, 19:7-131.

33. Barber BE, Grigg MJ, William T, Yeo TW, Anstey NM: The Treatment of Plasmodium knowlesi Malaria. Trends Parasitol 2017, 33:242-253.

34. Rajahram GS, Cooper DJ, William T, Grigg MJ, Anstey NM, Barber BE: Deaths from Plasmodium knowlesi malaria: Case series and systematic review. Clin Infect Dis 2019.

35. Collins WE: Plasmodium knowlesi: a malaria parasite of monkeys and humans. Annu Rev Entomol 2012, 57:107-121.

36. Pasini EM, Zeeman AM, Voorberg VANDERWA, Kocken CHM: Plasmodium knowlesi: a relevant, versatile experimental malaria model. Parasitology 2018, 145:56-70.

37. Galinski MR, Lapp SA, Peterson MS, Ay F, Joyner CJ, KG LER, Fonseca LL, Voit EO, MaHPIC C: Plasmodium knowlesi: a superb in vivo nonhuman primate model of antigenic variation in malaria. Parasitology 2018, 145:85-100. 
38. Knowles R, Gupta BMD: A Study of Monkey-Malaria, and Its Experimental Transmission to Man. Ind Med Gaz 1932, 67:301-320.

39. Coatney GR, Collins WE, Warren M, Contacos PG: The Primate Malarias. Washington, DC: U.S Department of Health, Education and Welfare; 1971.

40. Coatney GR, Collins WE, Warren M, Contacos PG: The Primate Malarias, e-book [original book published in 1971]

pp. 381. Atlanta, GA, USA: Division of Parasitic Diseases, Centers for Disease Control and Protection; 2003:381.

41. Brown $\mathrm{KN}$, Brown IN: Immunity to malaria: antigenic variation in chronic infections of Plasmodium knowlesi. Nature 1965, 208:1286-1288.

42. Butcher GA, Cohen S: Antigenic variation and protective immunity in Plasmodium knowlesi malaria. Immunology 1972, 23:503-521.

43. Barnwell JW, Howard RJ, Coon HG, Miller LH: Splenic requirement for antigenic variation and expression of the variant antigen on the erythrocyte membrane in cloned Plasmodium knowlesi malaria. Infect Immun 1983, 40:985-994.

44. Miller LH, Fremount HN, Luse SA: Deep vascular schizogony of Plasmodium knowlesi in Macaca mulatta. Distribution in organs and ultrastructure of parasitized red cells. Am J Trop Med Hyg 1971, 20:816-824.

45. Spangler WL, Gribble D, Abildgaard C, Harrison J: Plasmodium knowlesi malaria in the Rhesus monkey. Vet Pathol 1978, 15:83-91.

46. Butcher GA, Mitchell GH, Cohen S: Plasmodium knowlesi infections in a small number of non-immune natural hosts (Macaca fascicularis) and in rhesus monkeys (M. mulatta). Trans R Soc Trop Med Hyg 2010, 104:75-77.

47. Butcher GA: Models for malaria: Nature knows best. Parasitol Today 1996, 12:378382.

48. Anderios F, Noorrain A, Vythilingam I: In vivo study of human Plasmodium knowlesi in Macaca fascicularis. Exp Parasitol 2010, 124:181-189.

49. Barber BE, Russell B, Grigg MJ, Zhang R, William T, Amir A, Lau YL, Chatfield MD, Dondorp AM, Anstey NM, Yeo TW: Reduced red blood cell deformability in Plasmodium knowlesi malaria. Blood Adv 2018, 2:433-443.

50. Percie du Sert N, Hurst V, Ahluwalia A, Alam S, Avey MT, Baker M, Browne WJ, Clark A, Cuthill IC, Dirnagl U, et al: The ARRIVE guidelines 2.0: Updated guidelines for reporting animal research. $P$ LoS Biol 2020, 18:e3000410.

51. Lapp SA, Geraldo JA, Chien JT, Ay F, Pakala SB, Batugedara G, Humphrey J, Consortium M, De BJ, Le Roch KG, et al: PacBio assembly of a Plasmodium knowlesi genome sequence with $\mathrm{Hi}-\mathrm{C}$ correction and manual annotation of the SICAvar gene family. Parasitology 2018, 145:71-84.

52. Joyner CJ, Moreno A, Meyer EVS, Cabrera-Mora M, Consortium M, Kissinger JC, Barnwell JW, Galinski MR: Plasmodium cynomolgi infections in rhesus macaques display clinical and parasitological features pertinent to modelling vivax malaria pathology and relapse infections. Malar J 2016, 15:1-18.

53. Earle WC, Perez M: Enumeration of parasites in the blood of malarial patients. $J$ Lab Clin Med 1932, 17:1124-1130.

54. Fonseca LL, Joyner CJ, MaHPIC-Consortium, Galinski MR, Voit EO: A model of Plasmodium vivax concealment based on Plasmodium cynomolgi infections in 
55. Peterson MS, Joyner CJ, Cordy RJ, Salinas JL, Machiah D, Lapp SA, Consortium TM, Meyer VS, Gumber S, Galinski MR: Plasmodium vivax parasite load is associated with histopathology in Saimiri boliviensis with findings comparable to $P$. vivax pathogenesis in humans. Open Forum Infectious Diseases 2019, 6.

56. Tang Y, Joyner C, Cabrera-Mora M, Lapp S, Nural M, Pakala S, DeBarry J, Soderberg S, consortium M, Kissinger $\mathrm{J}$, et al: Integrative analysis associates monocytes with insufficient erythropoiesis during acute Plasmodium cynomolgi malaria in rhesus macaques. Malar J 2017, 16:1-16.

57. Fonseca LL, Alezi HS, Moreno A, Barnwell JW, Galinski MR, Voit EO: Quantifying the removal of red blood cells in Macaca mulatta during a Plasmodium coatneyi infection. Malar J 2016, 15:410.

58. Fonseca LL, Joyner CJ, Saney CL, MaHPIC-Consortium, Moreno A, Barnwell JW,

59. Fonseca LL, Voit EO: Comparison of mathematical frameworks for modeling

60. Moreno A, Cabrera-Mora M, Garcia A, Orkin J, Strobert E, Barnwell JW, Galinski MR: Plasmodium coatneyi in rhesus macaques replicates the multisystemic dysfunction of severe malaria in humans. Infect Imm 2013, 81:1889-1904.

61. Aurrecoechea C, Brestelli J, Brunk BP, Dommer J, Fischer S, Gajria B, Gao X, Gingle A, Grant G, Harb OS, et al: PlasmoDB: a functional genomic database for malaria parasites. Nucleic Acids Res 2009, 37:D539-543.

62. Mitchell GH, Butcher GA, Cohen S: Isolation of blood-stage merozoites from Plasmodium knowlesi malaria. Int J Parasitol 1973, 3:443-445.

63. Cordy RJ, Patrapuvich R, Lili LN, Cabrera-Mora M, Chien J-T, Tharp GK, Khadka M, Meyer EVS, Lapp SA, Joyner CJ, et al: Distinct amino acid and lipid perturbations characterize acute versus chronic malaria. JCI Insight 2019, 4.

64. Lacerda MV, Mourao MP, Coelho HC, Santos JB: Thrombocytopenia in malaria: who cares? Mem Inst Oswaldo Cruz 2011, 106 Suppl 1:52-63.

65. Perkins DJ, Were T, Davenport GC, Kempaiah P, Hittner JB, Ong'echa JM: Severe malarial anemia: innate immunity and pathogenesis. Int J Biol Sci 2011, 7:14271442.

66. Lamikanra AA, Brown D, Potocnik A, Casals-Pascual C, Langhorne J, Roberts DJ: Malarial anemia: of mice and men. Blood 2007, 110:18-28.

67. Chang $\mathrm{KH}$, Tam M, Stevenson MM: Inappropriately low reticulocytosis in severe malarial anemia correlates with suppression in the development of late erythroid precursors. Blood 2004, 103:3727-3735.

68. Thawani N, Tam M, Bellemare MJ, Bohle DS, Olivier M, de Souza JB, Stevenson MM: Plasmodium products contribute to severe malarial anemia by inhibiting erythropoietin-induced proliferation of erythroid precursors. J Infect Dis 2014, 209:140-149.

69. Kho S, Barber BE, Johar E, Andries B, Poespoprodjo JR, Kenangalem E, Piera KA, Ehmann A, Price RN, William T, et al: Platelets kill circulating parasites of all major Plasmodium species in human malaria. Blood 2018, 132:1332-1344. 
1219 70. Raja AI, Brickley EB, Taaffe J, Ton T, Zhao Z, Bock KW, Orr-Gonzalez S, Thomas ML, 3rd, Lambert LE, Moore IN, Duffy PE: A primate model of severe malarial anaemia: a comparative pathogenesis study. Sci Rep 2019, 9:18965.

71. White NJ: Anaemia and malaria. Malar J 2018, 17:371.

72. Collins WE, Jeffery GM, Roberts JM: A retrospective examination of anemia during infection of humans with Plasmodium vivax. Am J Trop Med Hyg 2003, 68:410-412.

73. Jakeman GN, Saul A, Hogarth WL, Collins WE: Anaemia of acute malaria infections in non-immune patients primarily results from destruction of uninfected erythrocytes. Parasitology 1999, 119 ( Pt 2):127-133.

74. Looareesuwan S, Merry AH, Phillips RE, Pleehachinda R, Wattanagoon Y, Ho M, Charoenlarp P, Warrell DA, Weatherall DJ: Reduced erythrocyte survival following clearance of malarial parasitaemia in Thai patients. Br J Haematol 1987, 67:473-478.

75. Knisely MH, Stratman-Thomas WK, et al.: Knowlesi malaria in monkeys; microscopic pathological circulatory physiology of rhesus monkeys during acute Plasmodium knowlesi malaria. J Natl Malar Soc 1945, 4:285-300.

1233

76. Miller LH, Usami S, Chien S: Alteration in the rheologic properties of Plasmodium

1235 knowlesi--infected red cells. A possible mechanism for capillary obstruction. J Clin Invest 1971, 50:1451-1455.

1238

77. Rigdon RH, Stratman Thomas WK: A Study of the Pathological Lesions in P. knowlesi

79. Moreno A, Garcia A, Cabrera-Mora M, Strobert E, Galinski MR: Disseminated Infection in M. Rhesus Monkeys. Amer J Trop Med Hyg 1942, S1-22:329-339.

78. Joyner CJ, Consortium M, Wood JS, Moreno A, Garcia A, Galinski MR: Case report: Severe and complicated cynomolgi malaria in a rhesus macaque resulted in similar histopathological changes as those seen in human malaria. Am J Trop Med Hyg 2017, 97:548-555. intravascular coagulation complicated by peripheral gangrene in a rhesus macaque (Macaca mulatta) experimentally infected with Plasmodium coatneyi. Am J Trop Med Hyg 2007, 76:648-654.

80. Lombardini ED, Gettayacamin M, Turner GD, Brown AE: A Review of Plasmodium coatneyi-Macaque Models of Severe Malaria. Vet Pathol 2015, 52:998-1011.

81. Dankwa S, Lim C, Bei AK, Jiang RH, Abshire JR, Patel SD, Goldberg JM, Moreno Y, Kono M, Niles JC, Duraisingh MT: Ancient human sialic acid variant restricts an emerging zoonotic malaria parasite. Nat Commun 2016, 7:11187.

1254 Haemoglobinuria in Certain Species of Monkey. Ind Med Gaz 1932, 67:246-249.

83. Schmidt LH, Fradkin R, Harrison J, Rossan RN: Differences in the virulence of Plasmodium knowlesi for Macaca irus (fascicularis) of Philippine and Malayan origins. Am J Trop Med Hyg 1977, 26:612-622.

84. Collins WE, Skinner JC, Broderson JR, Filipski VK, Morris CM, Stanfill PS, Warren M: Susceptibility of Macaca fascicularis monkeys from Mauritius to different species of Plasmodium. J Parasitol 1992, 78:505-511.

85. DeBarry JD, Kissinger JC, Nural MV, Pakala SB, Humphrey JC, Meyer EVS, Cordy RJ, Cabrera-Mora M, Trippe ED, Aguilar JB, et al: Practical Recommendations for Supporting a Systems Biology Cyberinfrastructure. Data Science Journal 2020, 19:112. 
bioRxiv preprint doi: https://doi.org/10.1101/2021.06.28.448877; this version posted June 28, 2021. The copyright holder for this preprint (which was not certified by peer review) is the author/funder. All rights reserved. No reuse allowed without permission.

1265

1266

1267

1268

1269

1270

1271

1272

1273

1274

1275

1276

1277

1278

1279

1280

1281

1282

1283

1284

1285

1286

1287

1288

1289

1290

1291

1292

1293

1294 
a bioRxiv preprint/doi: https://doi.org/10.1101/2021.06.28.448877; this version posted June 28, 2021. The copyright hlder for this preprint (Which was not certified by peer review) is the author/funge\%All rights reserved. No reuse allowed without permission.

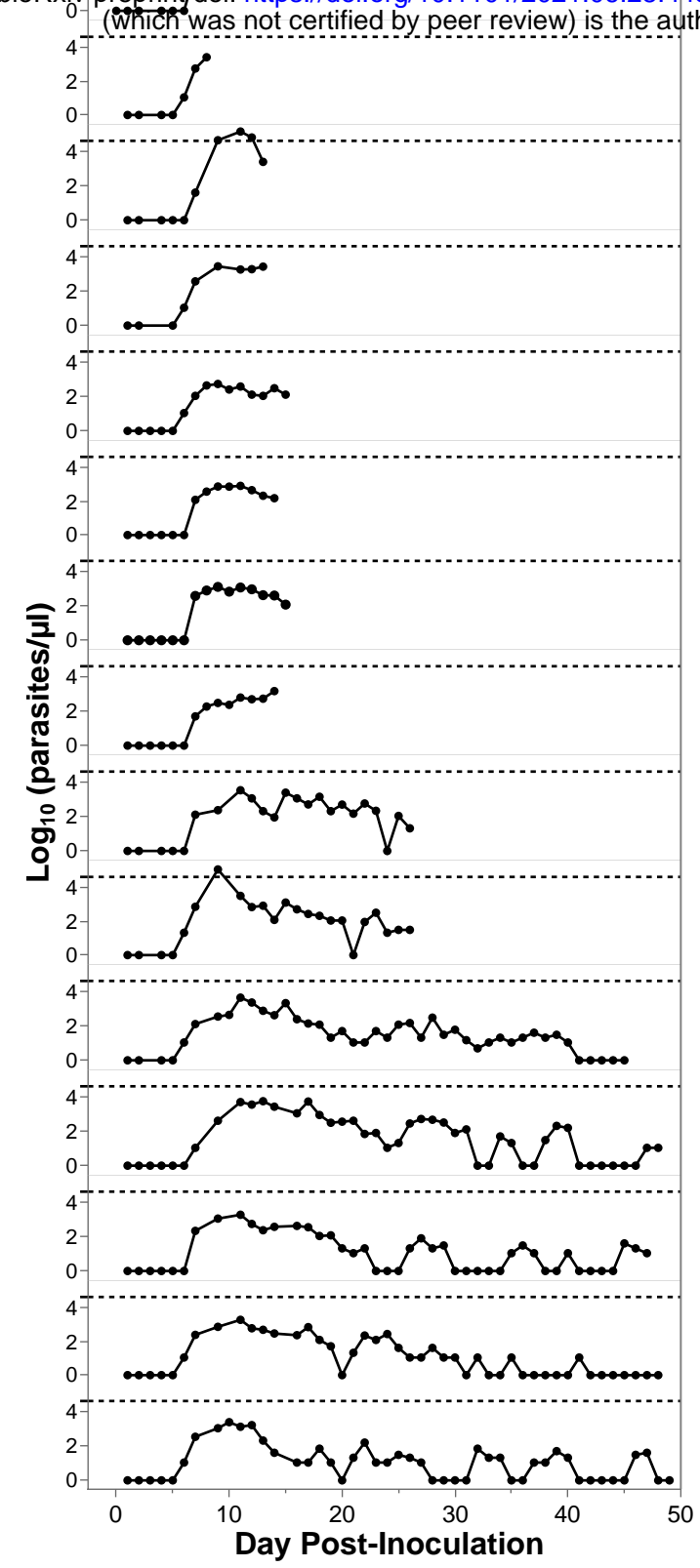

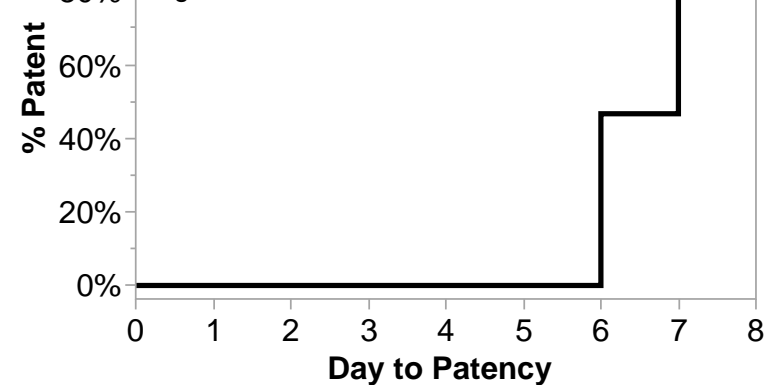

C

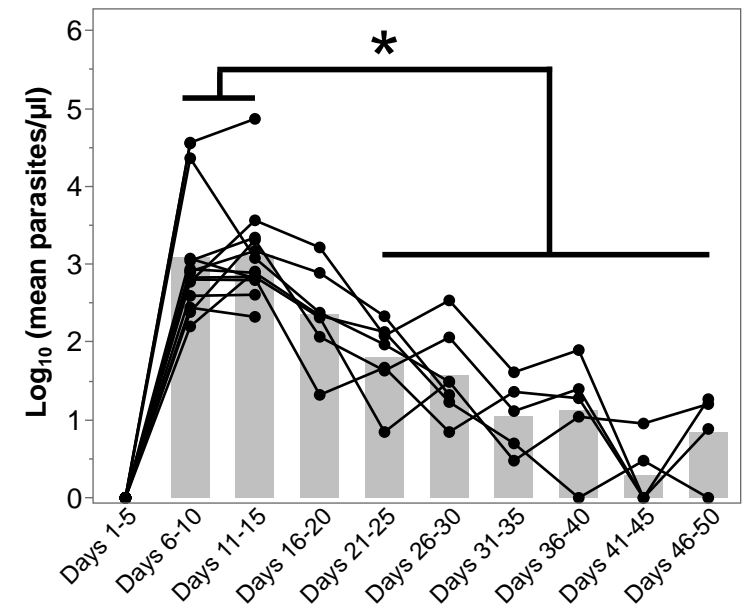


a

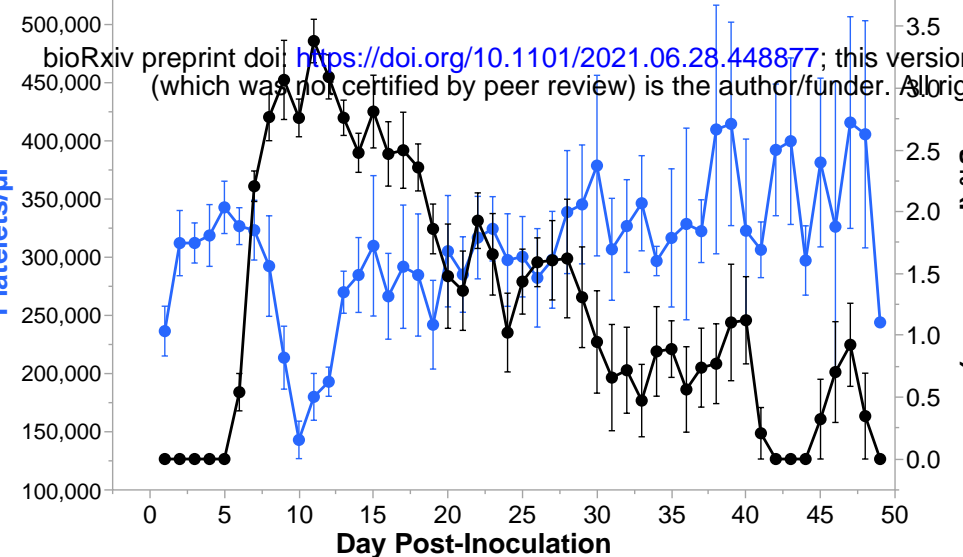

C

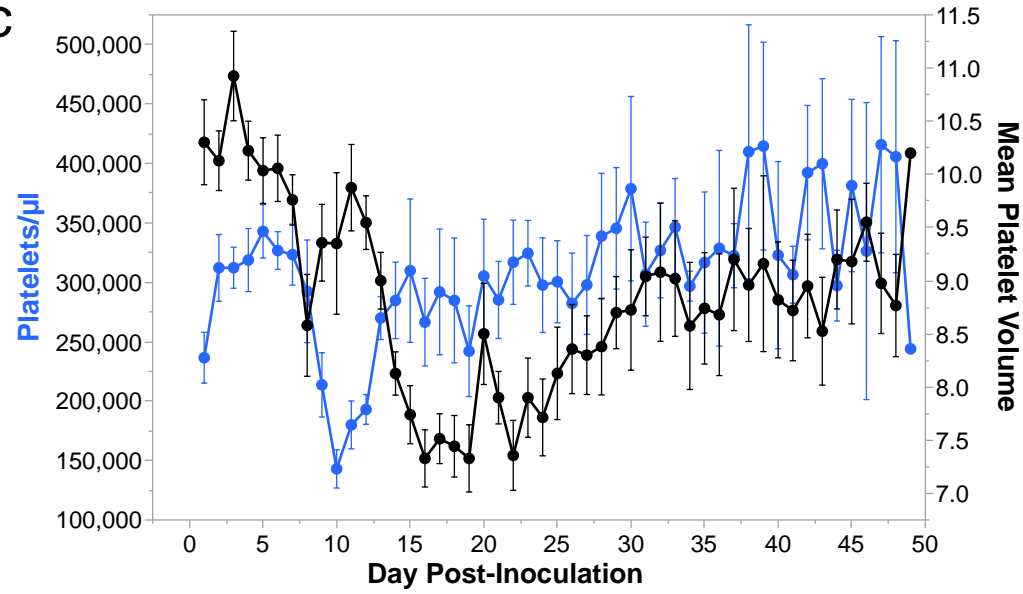

b

sion posted 6 GQnen028, 2021. The copyright holder for this preprint

- No reuse allowed without permission.

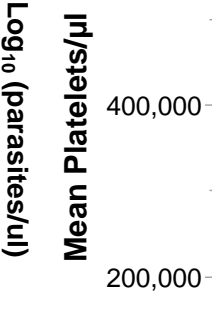

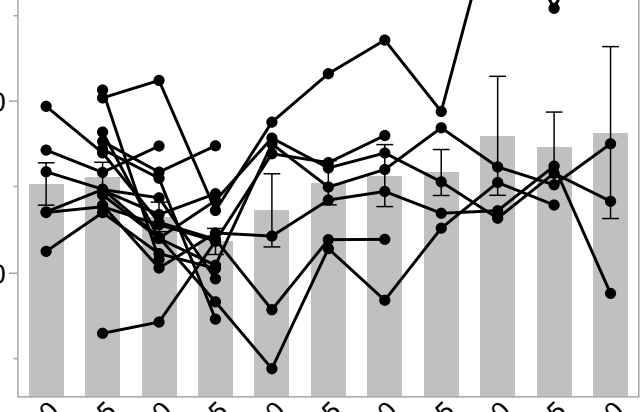

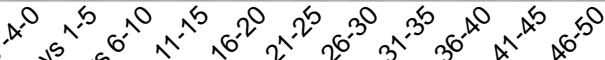

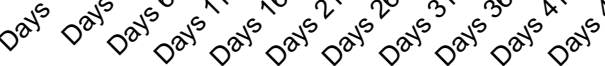


a bioRxiv preprint doi: https://doi.org/10.1101//2021.06.28.448877; this version posted June 28, 2021. The copyright holder for this preprint
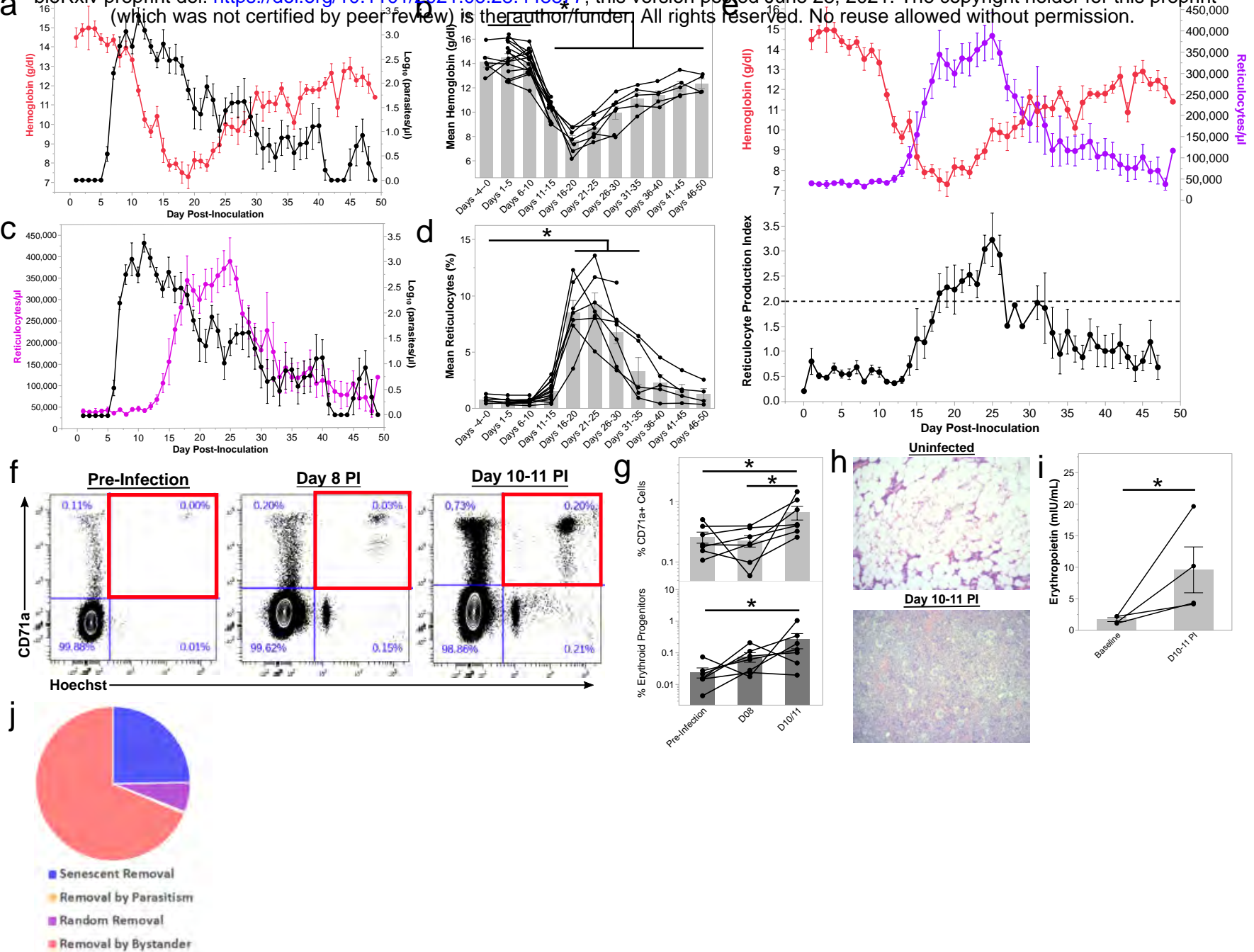
$\mathrm{a}^{40}$

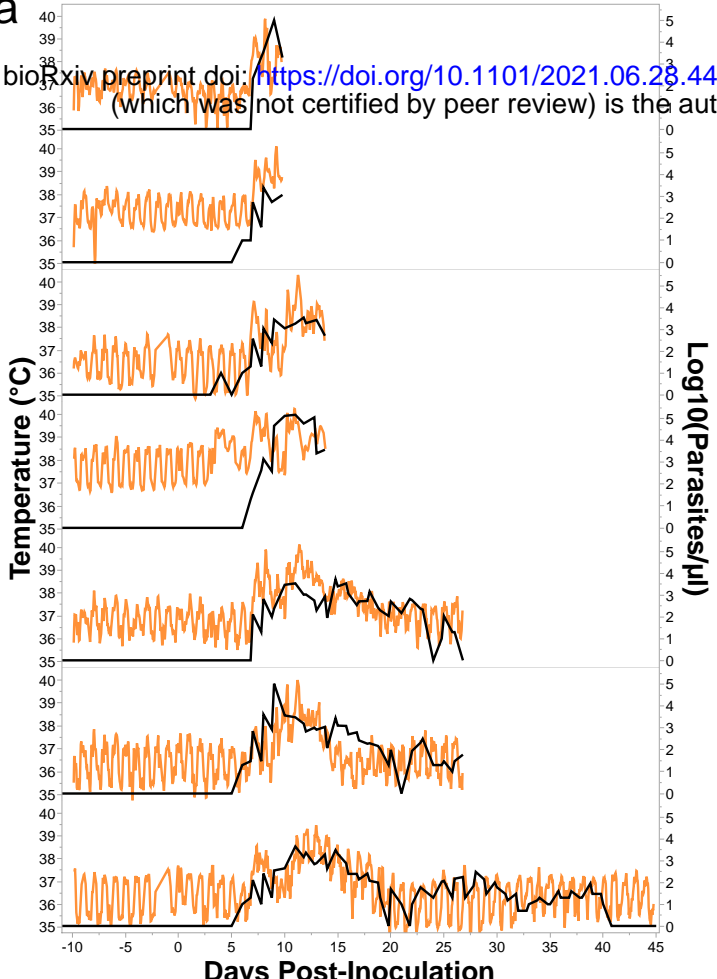

b

$100 \%$

7 ; thisoyersion posted June 28, 202 1 . The copyright holder for this preprint

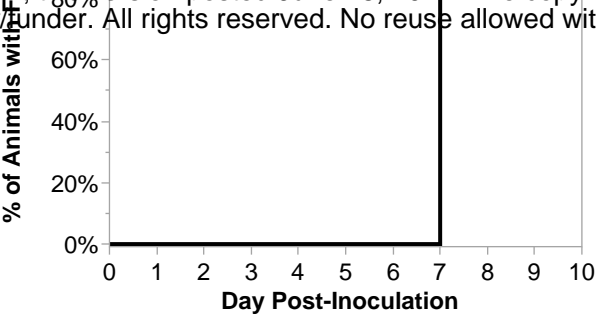

C

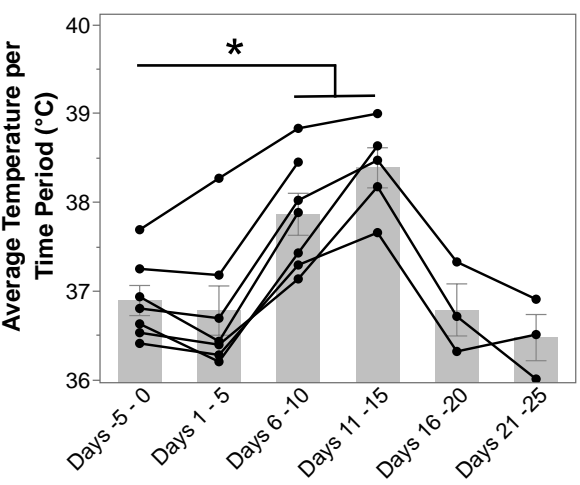


a Uninfected

Infected

b \& *

bioRxiv preprint dol: https//doi.org/10.1101/2021.06.28.448877; this versian posted June 28, 2021. The copyright holder for this preprint (which was not certified by peer review) is the author/funder. All rieğhts resefrved. No reuse allowed without permission.

Lung
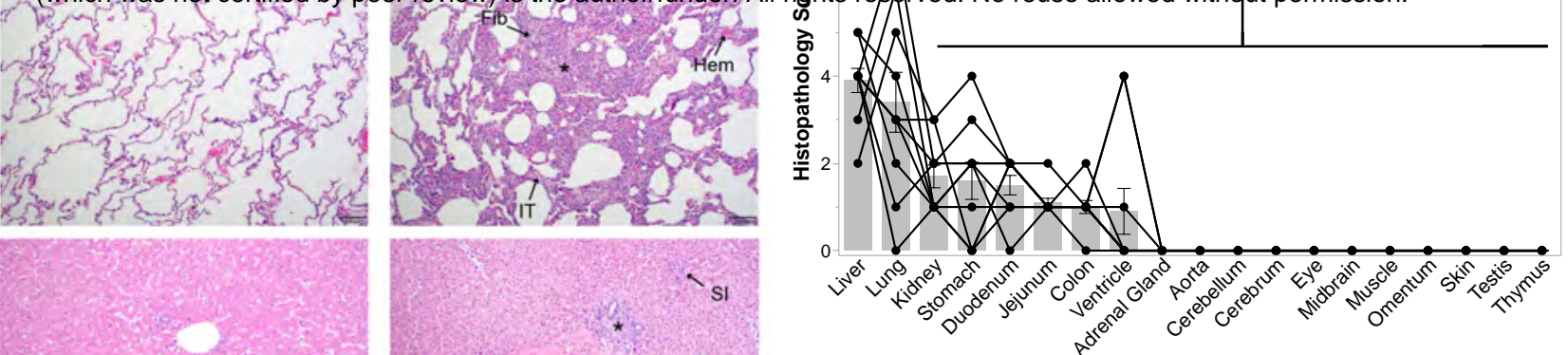

Liver
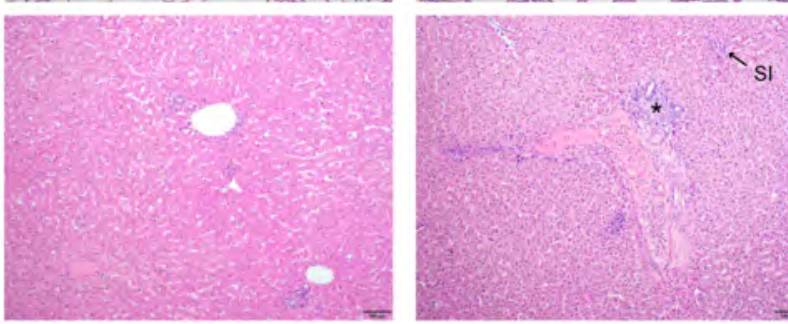

Kidney
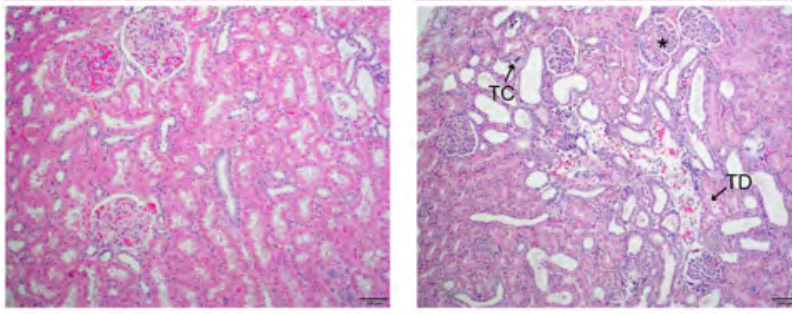

c

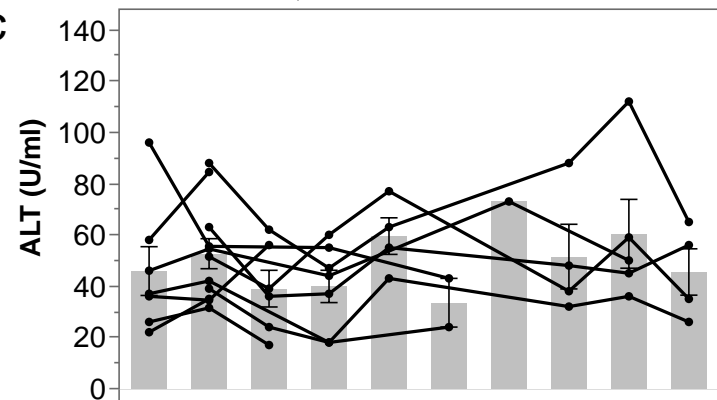

\section{Duodenum}

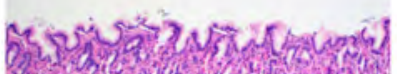

\section{Stomach}

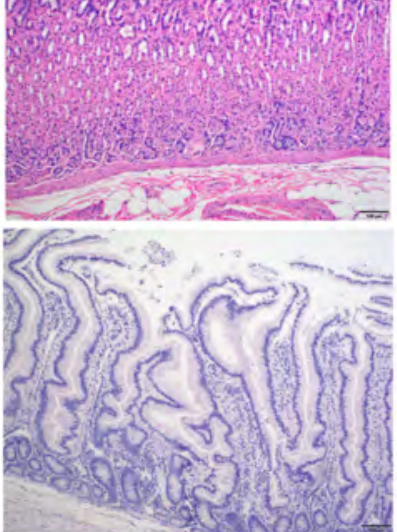

Jejunum
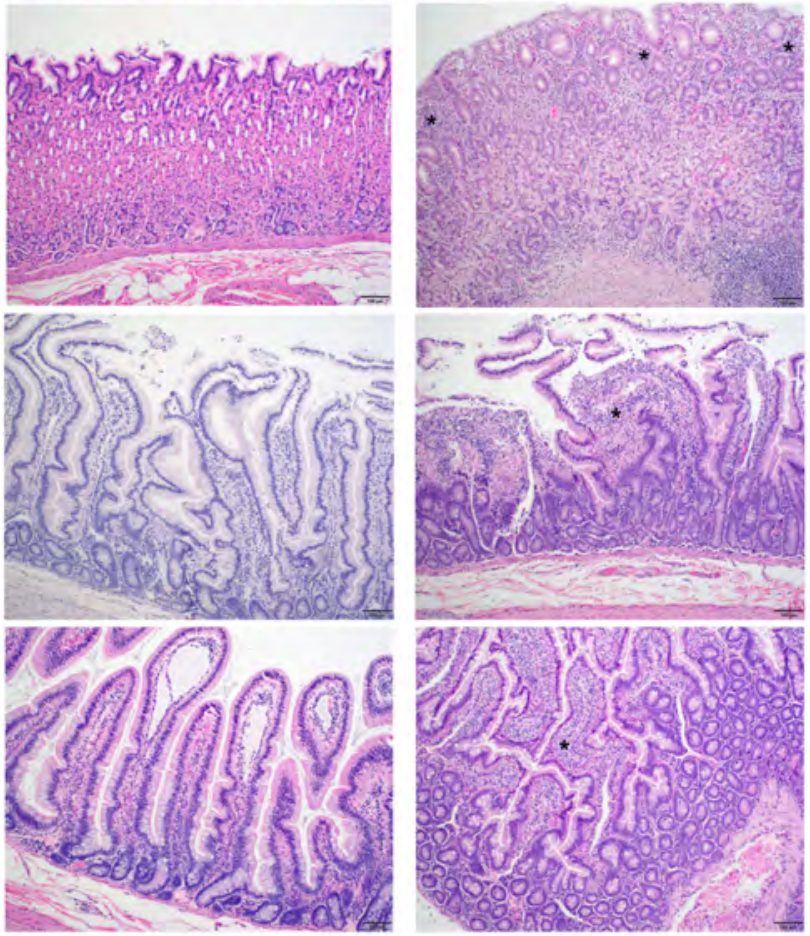

d

$$
60-
$$
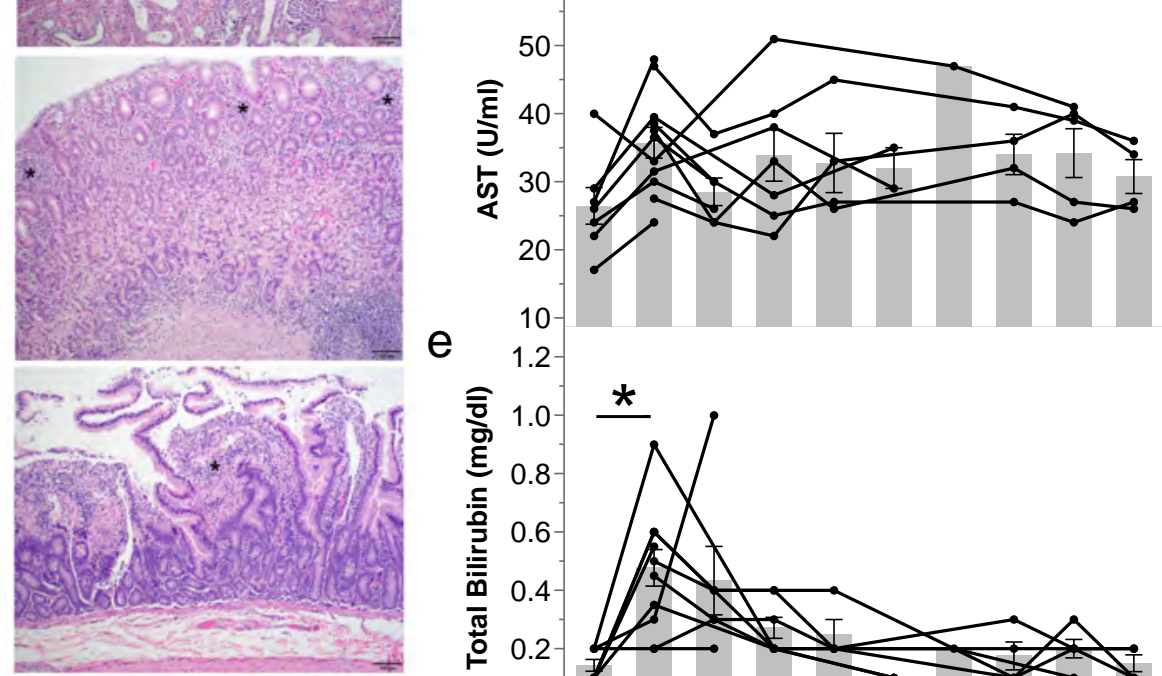

e $\quad 1.2$
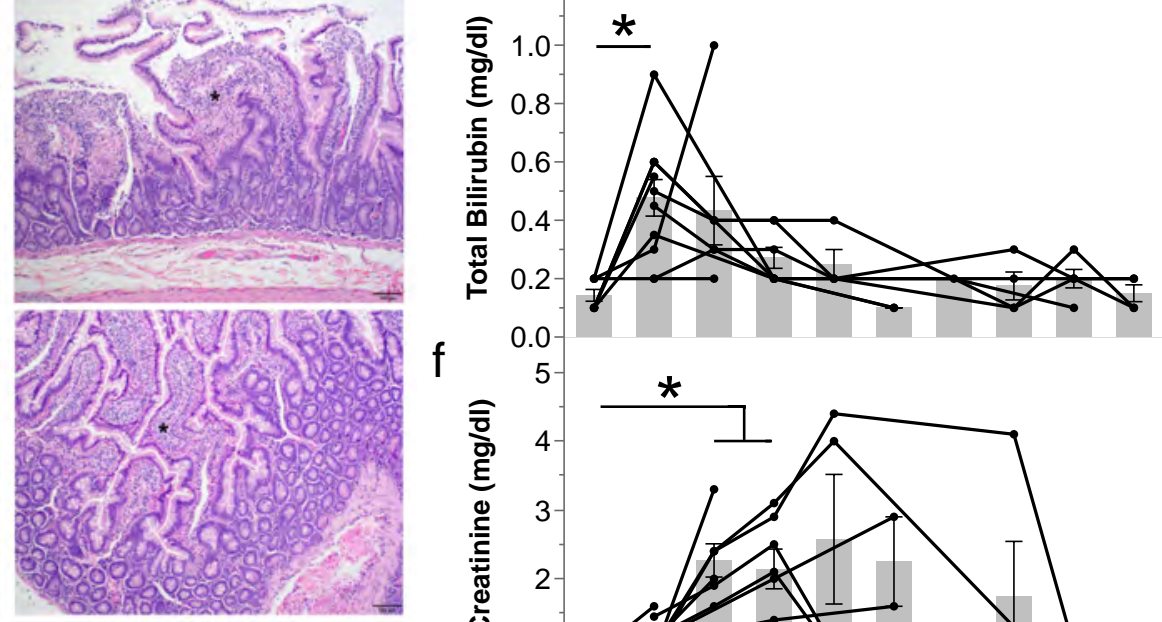

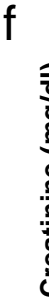

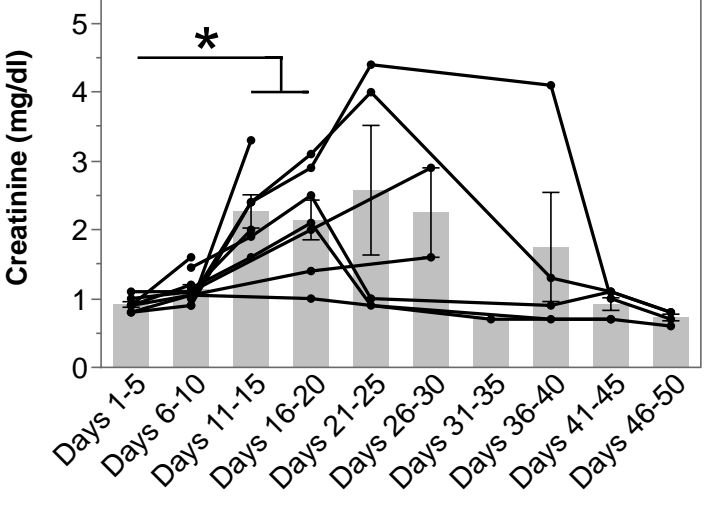

Colon

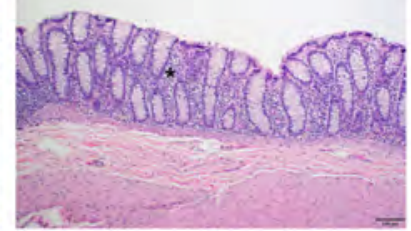




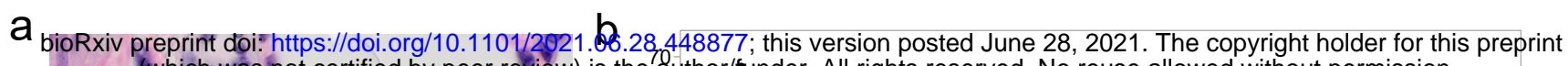
bioRxiv preprint doi: https://doi.org/10.1101/2021.
(which was hot certified by peer review) is the author/minder. All rights reserved. No reuse allowed without permission.
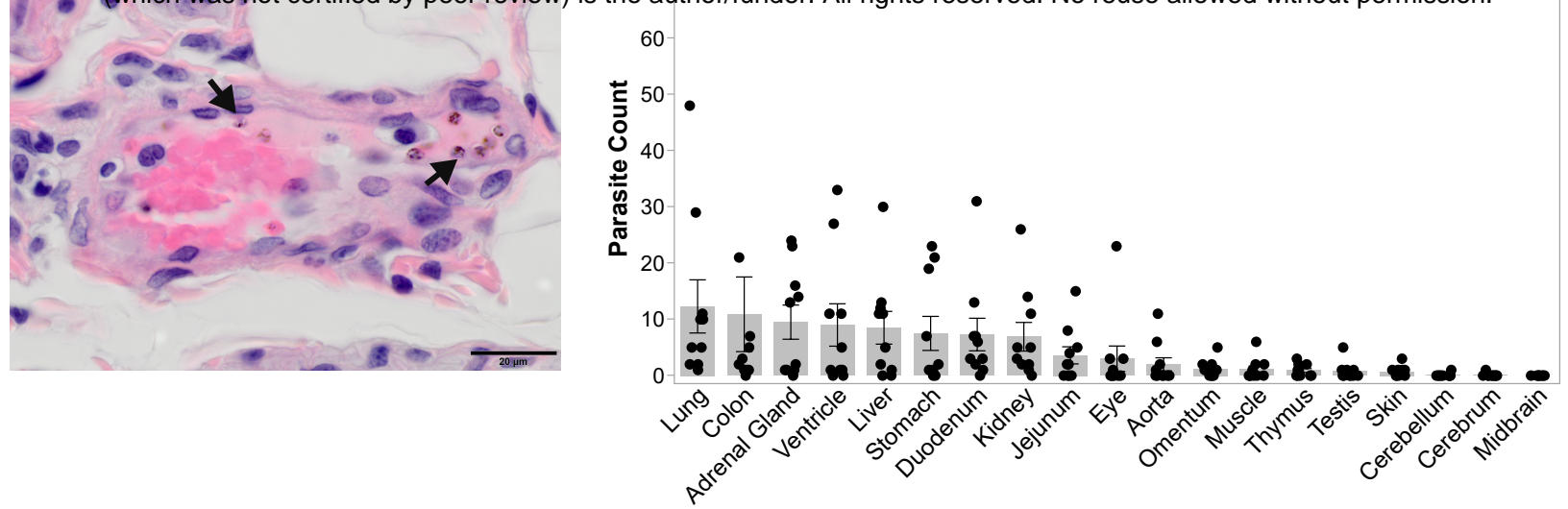